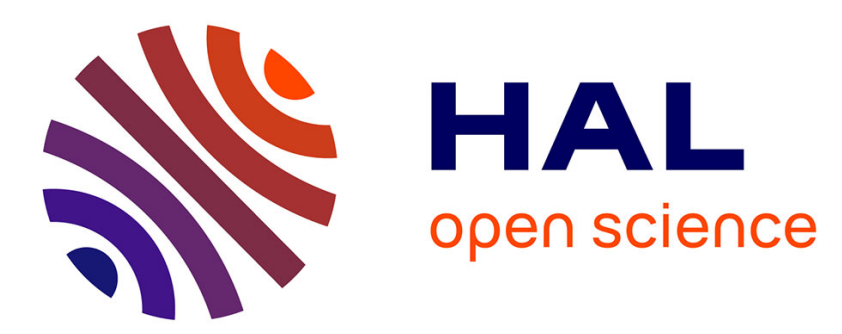

\title{
Stress rate formulation for elastoplastic models with internal variables based on augmented Lagrangian regularisation
}

Massimo Cuomo, Loredana Contrafatto

\section{- To cite this version:}

Massimo Cuomo, Loredana Contrafatto. Stress rate formulation for elastoplastic models with internal variables based on augmented Lagrangian regularisation. International Journal of Solids and Structures, 2000, 37, pp.3935 - 3964. hal-00913269

\section{HAL Id: hal-00913269 \\ https://hal.science/hal-00913269}

Submitted on 3 Dec 2013

HAL is a multi-disciplinary open access archive for the deposit and dissemination of scientific research documents, whether they are published or not. The documents may come from teaching and research institutions in France or abroad, or from public or private research centers.
L'archive ouverte pluridisciplinaire HAL, est destinée au dépôt et à la diffusion de documents scientifiques de niveau recherche, publiés ou non, émanant des établissements d'enseignement et de recherche français ou étrangers, des laboratoires publics ou privés. 


\title{
Stress rate formulation for elastoplastic models with internal variables based on augmented Lagrangian regularisation
}

\author{
M. Cuomo , L. Contrafatto \\ Istituto di Scienza delle Costruzioni, University of Catania, Facolta di Ingegneria, Viale A. Doria, 6, 95125, Catania, Italy'
}

\begin{abstract}
The constitutive laws of elasto plasticity with internal variables are described through the definition of suitable dual potentials, which include various bardening models. A family of variational principles for inelastic problems is obtained using convex analysis tools. The structural problem is analysed using the complementary energy (Prager Hodge) functional. The functional is regularised introducing an Augmented Lagrangian Regularisation for the indicator function of the elastic domain so that a smooth optimisation problem is obtained. In the numerical solution the discretised problem is reformulated in a finite step form using a fully implicit integration scheme and the functional is redefined in the space of the self equilibrated nodal stresses, after enforcing satisfaction of the equilibrium equations in a weak form. Numerical tests have shown good performance on the part of the algorithm, which approaches the converged solution for a considerably smaller number of elements as compared with other algorithms. The method is equally available for perfect or hardening plasticity.
\end{abstract}

Keysords: Plasticity; Stress formulation; Mixed F.E.M; Augmented Lagrangian

\section{Introduction}

Mixed methods have gained growing interest in computational literature, especially in the field of solid mechanics. They allow locking phenomena to be avoided (Olson, 1983), they offer better performance with distorted elements and guarantee faster convergence rates (Berkovic and Mijuca, 
1998). One of the main advantages of mixed formulations is therefore the possibility of using coarse meshes and low-order elements with a consequent reduction in computational effort.

After the pioneering work of Pian on assumed stress distributions (Pian and Sumihara, 1984), a major improvement was achieved following recognition of the equivalence of stress models to assumed displacement models (Simo and Rifai, 1990). Recent developments have extended the method to nonlinear problems (Weissmann, 1992; Weissman and Jamjian, 1993). These methods are based on the idea of deriving interpolations for the stress components that satisfy a priori the homogeneous part of the equilibrium equations. Simo et al. (1989) developed a method of analysis based on a mixed functional on the stresses, internal forces, plastic multipliers and displacements evaluated at the end of the load step, whose variation yields the equilibrium equations and the consistent return algorithm for evaluation of the stresses. The implementation of the algorithm given by the authors is not suitable for perfect plasticity.

The main goal of the present work is to develop a method for the incremental analysis of elasticplastic structures based on the complementary energy functional. Stress-based formulations have sometimes been used in limit analysis, but are not commonly employed for incremental analyses. It seems, however, that the formulation presents several computational advantages. First of all, the problem is set in the linear space of stress components, which is only required to be a subset of $\mathrm{L}^{2}$; moreover, stress components are bounded functions, whereas displacement components are generally not (actually, in the case of perfect plasticity they belong to the space of bounded deformations). Nonuniqueness of the displacement field is therefore not a problem for stress-based formulations, and this is very useful in the analysis of structures with vanishing tension (masonry-like materials) or compression (cable structure) resistance. In these cases, indeed, it is possible for undefined displacements to occur in those parts of the structure where zero stresses are present, while the remaining part of the structure is still able to take increments in load.

The method proposed here differs from those mentioned previously inasmuch as only discretised stresses are used as unknowns. The equilibrium equations are enforced by reformulating the problem in the space of the self-stresses, satisfying a priori the non-homogeneous part of the equilibrium equations. Stresses are therefore continuous across elements and are evaluated directly at nodes, avoiding projection procedures.

In order to obtain the relevant variational formulation of the problem, the elastic-plastic constitutive equations have been stated in terms of dual (convex) potentials and a family of variational principles has been derived embedding the plastic constraint in the stress potential. In the paper only small deformations are considered. The equilibrium conditions have been enforced in a weak sense starting from the generalised Hellinger-Reissner principle. The complementary energy functional thus obtained is non-regular due to the presence of the indicator function of the yield condition. An original method of regularisation is proposed, based on Augmented Lagrangian techniques, that have proved to be highly efficient in unilateral problems (Cuomo and Ventura, 1998).

The main purposes of the work, therefore, can be summarised as follows:

- to present a variational formulation of plastic constitutive models in the context of internal variables, using the tools of convex analysis; the plastic flow rule follows directly from Prager's consistency condition;

- to derive a generalisation of variational principles for the case examined;

- to apply consistent Augmented Lagrangian Regularisation procedures to obtain smooth saddle point problems;

- to implement a numerical algorithm based on stress interpolations and on reduction of the unknowns to the elements of the kernel of the equilibrium operator.

An outline of the paper follows. The elastic-plastic constitutive equations, including some forms of 
hardening models, are derived in section 2. In section 3 variational principles for rate and finite-step formulations are given and the structural problem is defined. The regularisation technique is also illustrated. The finite-element discretisation of the complementary variational formulation is described in section 4 and the numerical procedure in section 5. In section 6 the numerical efficiency of the method is illustrated through a classical example and comparisons are made with other methods. Section 7 ends the paper with some considerations.

\section{Elastic-plastic constitutive model}

\subsection{State variables}

Let us consider a solid occupying a region $B \subset \mathscr{R}^{3}$ and let $\partial B_{\mathrm{q}}$ and $\partial B_{\mathrm{u}}$ be the loaded and restrained parts of its boundary. The process is ruled by the following state variables, belonging to dual linear vector spaces:

$u \in U$ displacements

$\varepsilon \in D$ strains

$\alpha \in I$ kinematic internal variables $f \in U^{\prime}$ external forces

$\sigma \in D^{\prime}$ stresses

$\chi \in I^{\prime}$ thermodynamic forces

The internal variables are associated with the distortion mechanisms of the microstructure. It is assumed that no interaction occurs between macro and micro deformations.

For the sake of convenience the external forces $f$ will be split into $b$ (external forces defined in internal points of $B$ ), $q$ (surface traction defined on $\partial B_{\mathrm{q}}$ ) and $r$ (surface traction defined on $\partial B_{\mathrm{u}}$ ). The displacements in $B \cup \partial B_{\mathrm{q}}$ will be denoted with $u$, while $\bar{u}$ will indicate the displacements imposed on $\partial B_{\mathrm{u}}$.

In the following equations a dot will denote time differentiation. In the linear framework the velocity of deformations is thus simply $\dot{\varepsilon}$.

The external and internal virtual power are given by the duality pairing between dual variables:

$$
\begin{aligned}
& P_{\mathrm{e}}=\langle f, \dot{u}\rangle=\langle b, \dot{u}\rangle_{0}+\langle q, \dot{u}\rangle_{\partial B_{\mathrm{q}}}+\langle r, \dot{\bar{u}}\rangle_{\partial B_{\mathrm{u}}}=\int_{B} b \dot{u} \mathrm{~d} B+\int_{\partial B_{\mathrm{q}}} q \dot{u} \mathrm{~d} s+\int_{\partial B_{\mathrm{u}}} r \dot{\bar{u}} \mathrm{~d} s \quad \forall \dot{u} \in U, \quad \forall f \in U^{\prime} \\
& P_{i}=\langle\sigma, \dot{\varepsilon}\rangle=\int_{B} \sigma \dot{\varepsilon} \mathrm{d} B \quad \forall \dot{\varepsilon} \in D, \quad \forall \sigma \in D^{\prime} \\
& 0=\langle\chi, \dot{\alpha}\rangle=\int_{B} \chi \dot{\alpha} \mathrm{d} B \quad \forall \dot{\alpha} \in I, \quad \forall \chi \in I^{\prime}
\end{aligned}
$$

where $\langle,\rangle_{0}$ is the inner product in $\mathrm{L}^{2}$ and $\langle,\rangle_{\partial B}$ is the duality pairing between trace spaces. The product between local variables is the appropriate scalar product.

The hypothesis of infinitesimal deformation implies additivity of the reversible and irreversible parts of strains and kinematic internal variables, denoted with the indexes 'e' and 'p', respectively:

$$
\varepsilon=\varepsilon_{\mathrm{e}}+\varepsilon_{\mathrm{p}}
$$


$\alpha=\alpha_{\mathrm{e}}+\alpha_{\mathrm{p}}=0$

The latter equality stems from the fulfilment of (1) for every volume element.

The structural problem is defined by the following set of equations:

1. compatibility equations $C u=\varepsilon$;

2. equilibrium equations $C^{\prime} \sigma=f$.

$C: U \rightarrow D$ and $C^{\prime}: D^{\prime} \rightarrow U^{\prime}$ are (linear) adjoint compatibility and equilibrium operators.

3. Constitutive equations that describe the reversible and irreversible behaviour.

\subsection{Reversible behaviour}

Let $F(\varepsilon, \alpha, T)$ be the Helmoltz specific free energy functional. According to the generalised standard material hypothesis of Halphen and Nguyen (1975), it is assumed that locally $F$ is given by the sum of two lower semicontinuous convex potentials, depending, respectively, on the elastic deformations $\varepsilon_{\mathrm{e}}$ and on the internal variables $\alpha_{\mathrm{e}}$ only, i.e.

$$
F\left(\varepsilon_{\mathrm{e}}, \alpha_{\mathrm{e}}, T\right)=\varphi\left(\varepsilon_{\mathrm{e}}, T\right)+\pi\left(\alpha_{\mathrm{e}}, T\right)
$$

where $\varphi$ is the elastic potential and $\pi$ is the hardening potential. They are in general non-differentiable and accordingly the generalised elastic relations are given by:

$$
\left\{\begin{array}{l}
\sigma \in \partial \varphi\left(\varepsilon_{\mathrm{e}}\right) \\
\chi \in \partial \pi\left(\alpha_{\mathrm{e}}\right)
\end{array} ; \quad\left\{\begin{array}{l}
\varepsilon_{\mathrm{e}} \in \partial \varphi^{\prime}(\sigma) \\
\alpha_{\mathrm{e}} \in \partial \pi^{\prime}(\chi)
\end{array}\right.\right.
$$

In (2) $\varphi^{\prime}$ and $\pi^{\prime}$ are the conjugate potentials in Fenchel's sense (Rockafellar, 1970):

$$
\varphi^{\prime}(\sigma)=\sup _{\varepsilon_{\mathrm{e}} \in D}\left[\sigma \varepsilon_{\mathrm{e}}-\varphi\left(\varepsilon_{\mathrm{e}}\right)\right] \quad \pi^{\prime}(\chi)=\sup _{\alpha_{\mathrm{e}} \in I}\left[\chi \alpha_{\mathrm{e}}-\pi\left(\alpha_{\mathrm{e}}\right] .\right.
$$

\subsection{Irreversible behaviour}

The evolution of an irreversible process is governed by the maximum entropy principle. In the present case it leads to the Clausius-Duhem inequality

$$
\sigma \dot{\varepsilon}_{\mathrm{p}}+\chi \dot{\alpha}_{\mathrm{p}}-\frac{1}{T} \nabla T h \geq 0
$$

where $T$ is the temperature and $\mathrm{h}$ the heat flux. The dissipated power $D$, which is given by the sum of the power dissipated in the plastic deformation and the power dissipated as heat, is accordingly:

$$
D\left(\dot{\varepsilon}_{\mathrm{p}}, \dot{\alpha}_{\mathrm{p}}, T\right)=\sup _{(\sigma, \chi, h)}\left[\sigma \dot{\varepsilon}_{\mathrm{p}}+\chi \dot{\alpha}_{\mathrm{p}}-\frac{1}{T} \nabla T h\right]
$$

In the following paragraphs isothermal processes are considered, so the dependence on the variable $h$ vanishes.

It is assumed that the functional $D$ is a potential with the properties of being convex, proper, lower semicontinuous, positively homogeneous, and such that: 


$$
D\left(\dot{\varepsilon}_{\mathrm{p}}, \dot{\alpha}_{\mathrm{p}}\right): D \times I \rightarrow \bar{R}, \quad \bar{R}=R \cup\{+\infty\}, \quad\left\{\begin{array}{l}
D(0,0)=0 \\
D\left(\dot{\varepsilon}_{\mathrm{p}}, \dot{\alpha}_{\mathrm{p}}\right) \geq 0 \quad \forall\left(\dot{\varepsilon}_{\mathrm{p}}, \dot{\alpha}_{\mathrm{p}}\right) \in D \times I
\end{array}\right.
$$

This hypothesis leads to an associated constitutive law based on the existence of an elastic domain (Eve et al., 1990). Denoting by $K$ the set

$$
K=\left\{(\sigma, \chi) \in D^{\prime} \times I^{\prime}: \sigma \dot{\varepsilon}_{\mathrm{p}}+\chi \dot{\alpha}_{\mathrm{p}} \leq D\left(\dot{\varepsilon}_{\mathrm{p}}, \dot{\alpha}_{\mathrm{p}}\right) \quad \forall\left(\dot{\varepsilon}_{\mathrm{p}}, \dot{\alpha}_{\mathrm{p}}\right) \in D \times I\right\}
$$

it results that:

1. $D$ is the support function of $K$, i.e.

$$
D\left(\dot{\varepsilon}_{\mathrm{p}}, \dot{\alpha}_{\mathrm{p}}\right)=\operatorname{supp} K=\sup _{(\sigma, \chi) \in K}\left[\sigma \dot{\varepsilon}_{\mathrm{p}}+\chi \dot{\alpha}_{\mathrm{p}}\right]
$$

2. $K$ is given by the subdifferential of $D$ at the origin:

$$
K=\partial D(0,0) .
$$

Indeed, by definition of subdifferential, it is:

$$
\partial D\left(\dot{\varepsilon}_{\mathrm{p}}, \dot{\alpha}_{\mathrm{p}}\right)=\left\{\begin{array}{l}
(\sigma, \chi) \in D^{\prime} \times I^{\prime}: \\
\sigma\left(\tilde{\dot{\varepsilon}}_{\mathrm{p}}-\dot{\varepsilon}_{\mathrm{p}}\right)+\chi\left(\tilde{\dot{\alpha}}_{\mathrm{p}}-\dot{\alpha}_{\mathrm{p}}\right) \leq D\left(\tilde{\dot{\varepsilon}}_{\mathrm{p}}, \tilde{\dot{\alpha}}_{\mathrm{p}}\right)-D\left(\dot{\varepsilon}_{\mathrm{p}}, \dot{\alpha}_{\mathrm{p}}\right) \\
\forall\left(\tilde{\dot{\varepsilon}}_{\mathrm{p}}, \tilde{\dot{\alpha}}_{\mathrm{p}}\right) \in D \times I
\end{array}\right\}
$$

Writing (4) for $\dot{\varepsilon}_{\mathrm{p}}=\dot{\alpha}_{\mathrm{p}}=0$ the definition of $K$ is recovered. Since from (3) the elements of $K$ are conjugated to a plastic deformation equal to zero, it can immediately be concluded that

3. $K$ is the elastic domain.

It is possible, through a Legendre transformation, to obtain the conjugate potential of $D$

$$
D^{\prime}(\sigma, \chi)=\sup _{\left(\dot{\varepsilon}_{\mathrm{p}}, \dot{\alpha}_{\mathrm{p}}\right)}\left[\sigma \dot{\varepsilon}_{\mathrm{p}}+\chi \dot{\alpha}_{\mathrm{p}}-D\left(\dot{\varepsilon}_{\mathrm{p}}, \dot{\alpha}_{\mathrm{p}}\right)\right]=\text { ind } K(\sigma, \chi)
$$

so that the conjugate variables $(\sigma, \chi)$ and $\left(\dot{\varepsilon}_{\mathrm{p}}, \dot{\alpha}_{\mathrm{p}}\right)$ are related by Fenchel's equality

$$
D\left(\dot{\varepsilon}_{\mathrm{p}}, \dot{\alpha}_{\mathrm{p}}\right)+D^{\prime}(\sigma, \chi)=\sigma \dot{\varepsilon}_{\mathrm{p}}+\chi \dot{\alpha}_{\mathrm{p}}
$$

which implies:

$$
(\sigma, \chi) \in \partial \operatorname{supp} K\left(\dot{\varepsilon}_{\mathrm{p}}, \dot{\alpha}_{\mathrm{p}}\right) \Leftrightarrow\left(\dot{\varepsilon}_{\mathrm{p}}, \dot{\alpha}_{\mathrm{p}}\right) \in \partial \text { ind } K(\sigma, \chi) .
$$

Eq. (6) is a generalisation of the normality rule of classical plasticity.

Irreversible behaviour can therefore be described by defining the dissipation functional or its conjugate. In the latter case the elastic domain can be specified by means of a convex yield function $g$ such that

$$
K=\{(\sigma, \chi): g(\sigma, \chi) \leq 0\}
$$

and the flow rule becomes:

$$
\left(\dot{\varepsilon}_{\mathrm{p}}, \dot{\alpha}_{\mathrm{p}}\right) \in N_{\mathrm{K}}^{(\sigma, \chi)} \equiv \partial \text { ind } K=\partial \text { ind } R[g(\sigma, \chi)] \partial g(\sigma, \chi)=\lambda \partial g(\sigma, \chi)
$$

where $N_{\mathrm{K}}^{(\sigma, \chi)}$ is the outward normal cone of $K$ at the point $(\sigma, \chi)$ defined as: 


$$
N_{\mathrm{K}}^{(\sigma, \chi)}=\left\{\left(\dot{\varepsilon}_{\mathrm{p}}, \dot{\alpha}_{\mathrm{p}}\right): \dot{\varepsilon}_{\mathrm{p}}(\tilde{\sigma}-\sigma)+\dot{\alpha}_{\mathrm{p}}(\tilde{\chi}-\chi) \leq 0, \quad \forall(\tilde{\sigma}, \tilde{\chi}) \in K\right\}
$$

and $R_{-}[g(\sigma, \chi)]$ is the set of non positive real values taken by $g$. The term $\partial$ ind $R_{-}[g(\sigma, \chi)]$ is equal to a non negative scalar $\lambda$ such that:

$$
\begin{array}{ll}
\lambda=0 \quad \text { if } & g(\sigma, \chi)<0 \\
\lambda \geq 0 & \text { if } \quad g(\sigma, \chi)=0 .
\end{array}
$$

Eqs. (7) are equivalent to the Kuhn-Tucker conditions:

$\lambda \geq 0, \quad g \leq 0, \quad \lambda g=0$.

\subsection{Rate elastic-plastic relations}

Introducing the tangent elastic and hardening potentials, the rates of the stresses and thermodynamic forces are given by:

$$
\begin{aligned}
& \dot{\sigma} \in \partial_{\dot{\varepsilon}_{\mathrm{e}}} \varphi^{\mathrm{t}}\left(\varepsilon_{\mathrm{e}}, \dot{\varepsilon}_{\mathrm{e}}\right) \\
& \dot{\chi} \in \partial_{\dot{\alpha}_{\mathrm{e}}} \pi^{\mathrm{t}}\left(\alpha_{\mathrm{e}}, \dot{\alpha}_{\mathrm{e}}\right) .
\end{aligned}
$$

If $\varphi\left(\varepsilon_{\mathrm{e}}\right)$ e $\pi\left(\alpha_{\mathrm{e}}\right)$ are twice Gateaux differentiable at any point, the tangent potentials coincide with the quadratic forms associated with the Hessian of the elastic potentials:

$$
\begin{aligned}
\varphi^{\mathrm{t}}\left(\varepsilon_{\mathrm{e}}, \dot{\varepsilon}_{\mathrm{e}}\right) & =\frac{1}{2} \nabla_{\varepsilon_{\mathrm{e}}}^{2} \varphi\left(\varepsilon_{\mathrm{e}}\right) \dot{\varepsilon}_{\mathrm{e}} \dot{\varepsilon}_{\mathrm{e}} \\
\pi^{\mathrm{t}}\left(\alpha_{\mathrm{e}}, \dot{\alpha}_{\mathrm{e}}\right) & =\frac{1}{2} \nabla_{\alpha_{\mathrm{e}}}^{2} \pi\left(\alpha_{\mathrm{e}}\right) \dot{\alpha}_{\mathrm{e}} \dot{\alpha}_{\mathrm{e}} .
\end{aligned}
$$

The rate form of the inelastic constitutive relation derives from the consistency condition whose generalisation in the present framework is (Romano et al., 1993):

$$
(\dot{\sigma}, \dot{\chi}) \in T_{\mathrm{K}}^{(\sigma, \chi)}
$$

where $T_{\mathrm{K}}$ is the tangent cone of the admissible stresses at the point $(\sigma, \chi)$ defined as

$$
T_{\mathrm{K}}^{(\sigma, \chi)}=\left\{(\dot{\sigma}(t), \dot{\chi}(t)): \dot{\sigma} \tilde{\dot{\varepsilon}}_{\mathrm{p}}+\dot{\chi} \tilde{\dot{\alpha}}_{\mathrm{p}} \leq 0 \quad \forall\left(\tilde{\dot{\varepsilon}}_{\mathrm{p}}, \tilde{\dot{\alpha}}_{\mathrm{p}}\right) \in N_{\mathrm{K}}^{(\sigma, \chi)}\right\}
$$

and the time derivatives in (9) must be understood as right derivatives, i.e.

$$
\dot{\sigma}=\left(\frac{\mathrm{d} \sigma}{\mathrm{d} t}\right)_{\Delta t \rightarrow 0^{+}} \quad \dot{\chi}=\left(\frac{\mathrm{d} \chi}{\mathrm{d} t}\right)_{\Delta t \rightarrow 0^{+}}
$$

since the functions $\sigma(t), \chi(t)$ are first-order discontinuous when the stress point reaches the boundary of $K$. Eq. (10) is satisfied for any $(\dot{\sigma}, \dot{\chi})$ if $N_{\mathrm{K}}(\sigma, \chi)=\{\emptyset\}$, that is, if $(\sigma, \chi) \in$ int $K$. If $(\sigma, \chi)$ lies on the boundary of $K$, (9) requires that the stress rate be internal to the tangent cone to $K$ (Fig. 1).

The loading-unloading condition requires that 


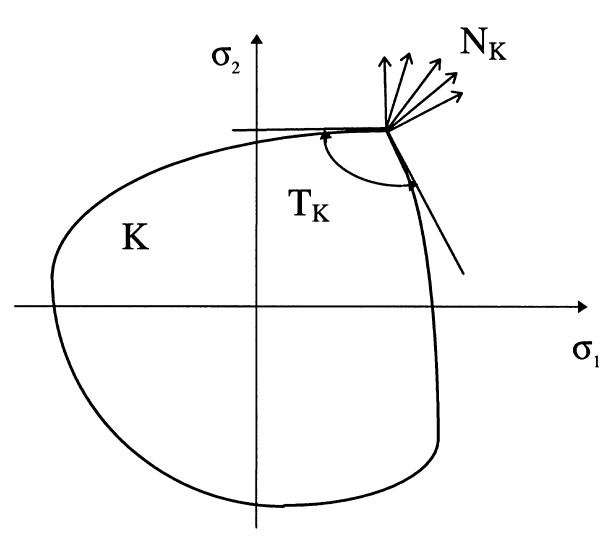

Fig. 1. Tangent cone to the admissible set $K$.

$$
\left(\dot{\varepsilon}_{\mathrm{p}}, \dot{\alpha}_{\mathrm{p}}\right) \in \partial\left[\operatorname{ind} T_{\mathrm{K}}^{(\sigma, \chi)}\right](\dot{\sigma}, \dot{\chi})
$$

that is, plastic deformations can develop only if $(\dot{\sigma}, \dot{\chi})$ lies on the boundary of $T_{\mathrm{K}}$.

It is easy to show that

$$
\partial \text { ind } T_{\mathrm{K}}^{(\sigma, \chi)}(\dot{\sigma}, \dot{\chi}) \subseteq N_{\mathrm{K}}^{(\sigma, \chi)}
$$

the equality holding at the origin $(\dot{\sigma}, \dot{\chi})=(0,0)$. Therefore consistency implies a more stringent condition on the flow rule.

For an admissible $(\dot{\sigma}, \dot{\chi})$ we have:

$$
\partial \text { ind } T_{\mathrm{K}}^{(\sigma, \chi)}=\left\{\left(\dot{\varepsilon}_{\mathrm{p}}, \dot{\alpha}_{\mathrm{p}}\right): \dot{\varepsilon}_{\mathrm{p}} \dot{\sigma}+\dot{\alpha}_{\mathrm{p}} \dot{\chi} \geq \dot{\varepsilon}_{\mathrm{p}} \tilde{\dot{\sigma}}+\dot{\alpha}_{\mathrm{p}} \tilde{\dot{\chi}}-\text { ind } T_{\mathrm{K}}(\tilde{\dot{\sigma}}, \tilde{\dot{\chi}}) \quad \forall(\tilde{\dot{\sigma}}, \tilde{\dot{\chi}}) \in D^{\prime} \times I^{\prime}\right\}
$$

that is, for the actual plastic deformation rates the following equality holds:

$$
\dot{\varepsilon}_{\mathrm{p}} \dot{\sigma}_{\mathrm{p}}+\dot{\alpha}_{\mathrm{p}} \dot{\chi}=\operatorname{supp} T_{\mathrm{K}}^{(\sigma, \chi)}=\operatorname{ind} T_{\mathrm{K}}^{0} \quad(\dot{\sigma}, \dot{\chi}) \in T_{\mathrm{K}}^{(\sigma, \chi)} .
$$

Eqs. (9) and (13) require that the dual variables $(\dot{\sigma}, \dot{\chi})-\left(\dot{\varepsilon}_{\mathrm{p}}, \dot{\alpha}_{\mathrm{p}}\right)$, belonging to polar cones, be related by an unilateral relationship, sketched in Fig. 2 in the uniaxial case. From the monotonicity of this relation it follows that ind $T_{\mathrm{K}}^{(\sigma, \chi)}$ and supp $T_{\mathrm{K}}^{(\sigma, \chi)}$ are conjugated potential functions:

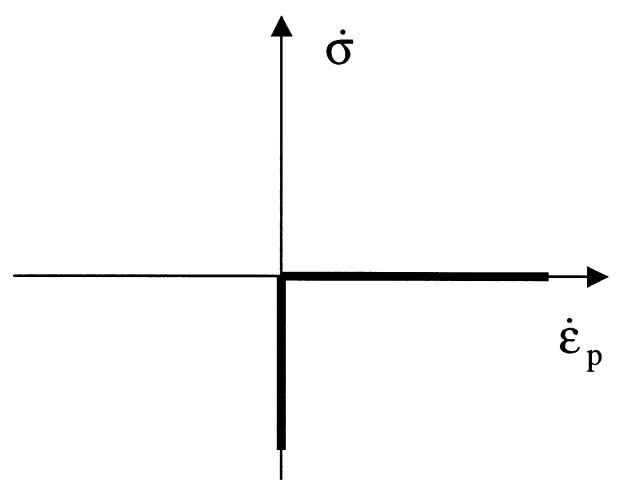

Fig. 2. Unilateral relationship between stress rate and plastic deformation rate. 


$$
V\left(\dot{\varepsilon}_{\mathrm{p}}, \dot{\alpha}_{\mathrm{p}}\right)=\operatorname{supp} T_{\mathrm{K}}^{(\sigma, \chi)}\left(\dot{\varepsilon}_{\mathrm{p}}, \dot{\alpha}_{\mathrm{p}}\right) \quad V^{\prime}(\dot{\sigma}, \dot{\chi})=\operatorname{ind} T_{\mathrm{K}}^{(\sigma, \chi)}(\dot{\sigma}, \dot{\chi})
$$

so that

$$
(\dot{\sigma}, \dot{\chi}) \in \partial\left[\operatorname{supp} T_{\mathrm{K}}^{(\sigma, \chi)}\right]\left(\dot{\varepsilon}_{\mathrm{p}}, \dot{\alpha}_{\mathrm{p}}\right)
$$

and Fenchel's inequality yields:

$$
V\left(\dot{\varepsilon}_{\mathrm{p}}, \dot{\alpha}_{\mathrm{p}}\right)+V^{\prime}(\dot{\sigma}, \dot{\chi}) \leq \dot{\sigma} \dot{\varepsilon}_{\mathrm{p}}+\dot{\chi} \dot{\alpha}_{\mathrm{p}} .
$$

If the pairs $(\dot{\sigma}, \dot{\chi})-\left(\dot{\varepsilon}_{\mathrm{p}}, \dot{\alpha}_{\mathrm{p}}\right)$ are conjugated the previous equation holds as an equality. It is a generalisation for non differentiable yield functions of Druker's inequality, stating that in the case of plasticity without internal variables including hardening effects

$$
\dot{\sigma} \dot{\varepsilon}_{\mathrm{p}} \geq 0 \text {. }
$$

The equality sign in (16) applies in perfect plasticity.

In the present formulation Eq. (16) is substituted by

$$
\dot{\sigma} \dot{\varepsilon}_{\mathrm{p}}+\dot{\chi} \dot{\alpha}_{\mathrm{p}}=0
$$

which follows immediately from (15) for admissible rates. Indeed, in the case $(\dot{\sigma}, \dot{\chi}) \in T_{\mathrm{K}}$ and $\left(\dot{\varepsilon}_{\mathrm{p}}, \dot{\alpha}_{\mathrm{p}}\right) \in$ $N_{\mathrm{K}}$ from (14) it follows that $V=V^{\prime}=0$. Condition (17) states that the direction of plastic flow is orthogonal to the rates $(\dot{\sigma}, \dot{\chi})$. Note that the rate formulation has the same structure as perfect unilateral contact relations.

If $g$ is differentiable at a point $(\sigma, \chi) \in \mathrm{bd} K$, i.e. such that $g(\sigma, \chi)=0$, an explicit representation of $T_{\mathrm{K}}$ is obtained:

$$
T_{\mathrm{K}}^{(\sigma, \chi)}=\{(\dot{\sigma}(t), \dot{\chi}(t)): \dot{g}(\sigma, \dot{\sigma}, \chi, \dot{\chi}) \leq 0, \quad g(\sigma, \chi)=0\}
$$

with $\dot{g}$ given by

$$
\dot{g}(\sigma, \dot{\sigma}, \chi, \dot{\chi}) \stackrel{\text { def }}{=} \nabla_{\sigma} g(\sigma, \chi) \dot{\sigma}+\nabla_{\chi} g(\sigma, \chi) \dot{\chi}
$$

so that

$$
\nabla_{(\dot{\sigma}, \dot{\gamma})} \dot{g}(\sigma, \chi)=\nabla_{(\sigma, \chi)} g(\sigma, \chi) .
$$

The flow rule (11) becomes:

$$
\begin{aligned}
& \left(\dot{\varepsilon}_{\mathrm{p}}, \dot{\alpha}_{\mathrm{p}}\right) \in N_{\mathrm{T}_{\mathrm{K}}}=\partial \text { ind } R[\dot{g}(\dot{\sigma}, \dot{\chi})] \partial \dot{g}(\dot{\sigma}, \dot{\chi})=\lambda \partial \dot{g}(\dot{\sigma}, \dot{\chi})=\lambda \nabla_{(\dot{\sigma}, \dot{\alpha})} \dot{g}(\sigma, \chi) \quad \text { if } \quad g=0 \\
& \left(\dot{\varepsilon}_{\mathrm{p}}, \dot{\alpha}_{\mathrm{p}}\right)=(0,0) \quad \text { if } \quad g<0 .
\end{aligned}
$$

This is equivalent to the Kuhn-Tucker conditions:

$$
\begin{aligned}
& \lambda=0 \quad \text { if } \dot{g}<0, \quad g=0 \\
& \lambda>0 \quad \text { if } \quad \dot{g}=0, \quad g=0 \\
& \lambda \dot{g}=0
\end{aligned}
$$


Conditions $(18,7)$ fully define the flow rule equations.

In the case of a corner point Kuhn-Tucker relations (18) hold for each yield surface:

$$
\lambda_{i}>0, \quad \dot{g}_{i} \leq 0, \quad \lambda_{i} \dot{g}_{i}=0
$$

where $g_{g}=0, i=1, \ldots, n$. Since convexity rules out the possibility of having an identical normal to two different yield surfaces, it follows that

$$
g_{i} \leq 0, \quad i=1, \ldots, n, \quad i \neq j, \quad \dot{g}_{j}=0
$$

so that only the $j$-th plastic mechanism will be activated.

\subsection{Some forms of tangent elastic and hardening potentials}

In this section we will state some forms of the free energy potential that will be used in the applications.

\subsubsection{The elastic tangent potential}

For the linear elastic potential $\varphi\left(\varepsilon_{\mathrm{e}}\right)$ Eq. (8) yields:

$$
\varphi^{\mathrm{t}}\left(\varepsilon_{\mathrm{e}}, \dot{\varepsilon}_{\mathrm{e}}\right)=\frac{1}{2} E \dot{\varepsilon}_{\mathrm{e}} \dot{\varepsilon}_{\mathrm{e}}
$$

where $E$ is the elastic tensor of the material, and the complementary elastic tangent potential is

$$
\varphi^{\prime t}(\sigma, \dot{\sigma})=\sup _{\dot{\varepsilon}_{\mathrm{e}} \in D}\left[\dot{\sigma} \dot{\varepsilon}_{\mathrm{e}}-\frac{1}{2} E \dot{\varepsilon}_{\mathrm{e}} \dot{\varepsilon}_{\mathrm{e}}\right]=\frac{1}{2} E{ }^{1} \dot{\sigma} \dot{\sigma}
$$

\subsubsection{The hardening tangent potential}

Isotropic, kinematic and mixed hardening are considered.

Let $\alpha_{1}$ and $\alpha_{2}$ be the internal variables, associated with kinematic and isotropic hardening, respectively, and $\chi_{1}$ and $\chi_{2}$ the dual thermodynamic forces. The yield function is written as:

$$
g(\sigma, \chi)=f\left(\sigma-\chi_{1}\right)-\left(k+\chi_{2}\right) \quad k \in R^{+}
$$

where $\chi_{1}$ is the back-stress tensor and $\chi_{2}$ is a scalar.

In particular for the Von Mises elastic domain, the yield function is given by the expression:

$$
g(\sigma, \chi)=\sqrt{\frac{3}{2}} J_{2}\left(\sigma-\chi_{1}\right)-\left(k+\chi_{2}\right)
$$

where $J_{2}=\operatorname{tr}\left[\operatorname{dev}\left(\sigma-\chi_{1}\right)\right]^{2}$ and $k=\sqrt{ } 3 / 2 J_{0}=\sigma_{0}, J_{0}$, being the second invariant of the deviatoric part of the stress tensor in the uniaxial case and $\sigma_{0}$ the tensile resistance of the material. In a plain stress state we have:

$$
J_{2}=\frac{2}{3}\left[\left(\sigma_{x}-\chi_{1 x}\right)^{2}+\left(\sigma_{y}-\chi_{1 y}\right)^{2}-\left(\sigma_{x}-\chi_{1 x}\right)\left(\sigma_{y}-\chi_{1 y}\right)+3\left(\tau_{x y}-\chi_{1 x y}\right)^{2}\right] .
$$

An additive form is adopted for the hardening potential

$$
\pi\left(\alpha_{\mathrm{e}}\right)=\pi_{1}\left(\alpha_{\mathrm{e}_{1}}\right)+\pi_{2}\left(\alpha_{\mathrm{e}_{2}}\right)
$$


and for each term the following expression is used (Simo and Taylor, 1985):

$$
\pi\left(\alpha_{\mathrm{e}}\right)=\frac{1}{2} H \alpha_{\mathrm{e}} \alpha_{\mathrm{e}}+c_{\infty}\left(\left\|\alpha_{\mathrm{e}}\right\|+\frac{1}{\eta} \mathrm{e}^{\eta\left\|\alpha_{\mathrm{e}}\right\|}\right) \quad c_{\infty}, \eta>0 .
$$

The first term describes a linear hardening with modulus $H$; the second introduces a transitory non linear hardening with initial modulus $c_{\infty} \eta$.

From constitutive Eqs. (2) the internal forces are given by:

$$
\begin{aligned}
& \chi_{1}=\nabla_{\alpha_{\mathrm{e}_{1}}} \pi_{1}=H_{1} \alpha_{\mathrm{e}_{1}}+\frac{c_{\infty_{1}}}{\left\|\alpha_{\mathrm{e}_{1}}\right\|}\left(1-\mathrm{e}^{\eta_{1}\left\|\alpha_{\mathrm{e}_{1}}\right\|}\right) \alpha_{\mathrm{e}_{1}} \\
& \chi_{2}=\nabla_{\alpha_{\mathrm{e}_{2}}} \pi_{2}=H_{2} \alpha_{\mathrm{e}_{2}}+\frac{c_{\infty_{2}}}{\left|\alpha_{\mathrm{e}_{2}}\right|}\left(1-\mathrm{e}^{\eta_{2}\left|\alpha_{\mathrm{e}_{2}}\right|}\right) \alpha_{\mathrm{e}_{2}} .
\end{aligned}
$$

The $\chi_{2}-\alpha_{\mathrm{e}}$, relation is shown in Fig. 3.

The tangent hardening modulus is

$$
H_{\mathrm{t}}=\nabla_{\alpha_{\mathrm{e}} \alpha_{\mathrm{e}}}^{2} \Pi\left(\alpha_{\mathrm{e}}\right)=H+\frac{c_{\infty}}{\left\|\alpha_{\mathrm{e}}\right\|^{2}}\left[\eta \mathrm{e}^{\eta\left\|\alpha_{\mathrm{e}}\right\|}-\frac{1}{\left\|\alpha_{\mathrm{e}}\right\|}\left(1-\mathrm{e}^{\eta\left\|\alpha_{\mathrm{e}}\right\|}\right)\right] \alpha_{\mathrm{e}} \otimes \alpha_{\mathrm{e}}+\frac{c_{\infty}}{\left\|\alpha_{\mathrm{e}}\right\|} \mathrm{e}^{\eta\left\|\alpha_{\mathrm{e}}\right\|}
$$

which, in the isotropic case, becomes:

$$
H_{\mathrm{t}}=H+c_{\infty} \mathrm{e}^{\eta\left|\alpha_{\mathrm{e}}\right|}
$$

The potential $\pi^{\prime t}(\chi, \dot{\chi})$ is

$$
\pi^{\prime \mathrm{t}}(\chi, \dot{\chi})=\frac{1}{2} H_{\mathrm{t}}{ }^{1}(\chi) \dot{\chi} \dot{\chi}
$$

where $H_{\mathrm{t}}^{-1}(\chi)$ is the inverse tangent hardening modulus. In the case of linear hardening the function $H_{\mathrm{t}}^{-1}(\chi)$ is immediate, e.g. in the isotropic case it is $H_{\mathrm{t}}=E E_{\mathrm{p}} /\left(E-E_{\mathrm{p}}\right)=$ constant; otherwise it can be obtained numerically as follows. First it is solved (19) w.r.t. $\alpha$. The solution is inserted in (20) and the inverse tangent hardening modulus is evaluated as:

$$
H_{\mathrm{t}}{ }^{1}=\left[\nabla_{\alpha_{\mathrm{e}} \alpha_{\mathrm{e}}}^{2} \Pi\left(\alpha_{\mathrm{e}}\right)\right]^{1} .
$$
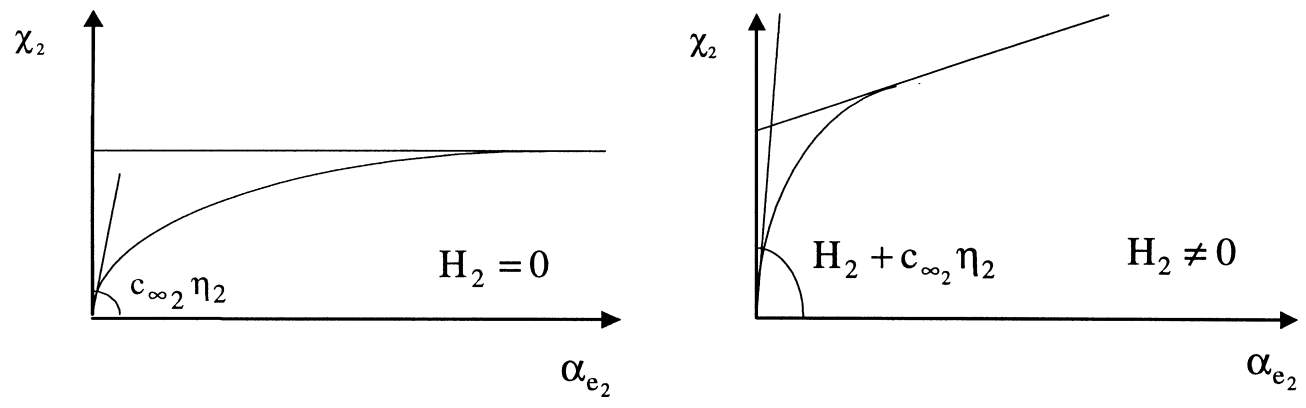

Fig. 3. Relation between $\chi_{2} \quad \alpha_{2}$ for isotropic non linear hardening. 


\section{Variational formulation}

\subsection{Rate formulation}

The rate form of the state equations for the structural problem under investigation obtained in section 2 is summarised as follows:

$$
\begin{aligned}
& \text { compatibility }\{C \dot{u}=\dot{\varepsilon} \\
& \text { equilibrium }\left\{C^{\prime} \dot{\sigma}=\dot{f}\right. \\
& \text { constitutive equations }\left\{\begin{array}{c}
\dot{\sigma} \in \partial_{\dot{\varepsilon}_{\mathrm{e}}} \varphi^{\mathrm{t}}\left(\varepsilon_{\mathrm{e}}, \dot{\varepsilon}_{\mathrm{e}}\right) \\
\dot{\chi} \in \partial_{\dot{\alpha}_{\mathrm{e}}} \pi^{\mathrm{t}}\left(\alpha_{\mathrm{e}}, \dot{\alpha}_{\mathrm{e}}\right) \\
\left(\dot{\varepsilon}_{\mathrm{p}}, \dot{\alpha}_{\mathrm{p}}\right) \in \partial\left[\text { ind } T_{\mathrm{K}}^{(\sigma, \chi)}\right](\dot{\sigma}, \dot{\chi}) \\
\dot{f} \in \partial_{\mathrm{u}} \gamma^{\mathrm{t}}(\dot{u})
\end{array}\right.
\end{aligned}
$$

under the hypothesis of additive decomposition for kinematic variables:

$$
\begin{aligned}
& \dot{\varepsilon}=\dot{\varepsilon}_{\mathrm{e}}+\dot{\varepsilon}_{\mathrm{p}} \\
& 0=\dot{\alpha}_{\mathrm{e}}+\dot{\alpha}_{\mathrm{p}} .
\end{aligned}
$$

It has been assumed that the rate of external forces can be obtained through a local tangent potential $\gamma^{\mathrm{t}}(\dot{u})$, which is concave in the case of convex boundary conditions.

The state Eqs. (21) are the stationarity conditions of the mixed functional (Romano and Alfano, 1995)

$$
\begin{aligned}
& \Omega\left(\dot{u}, \dot{f}, \dot{\varepsilon}_{\mathrm{e}}, \dot{\varepsilon}_{\mathrm{p}}, \dot{\alpha}_{\mathrm{e}}, \dot{\alpha}_{\mathrm{p}}, \dot{\sigma}, \dot{\chi}\right)\langle C \dot{u}, \dot{\sigma}\rangle+\Gamma^{\prime \mathrm{t}}(\dot{f})-\langle\dot{f}, \dot{u}\rangle-\left\langle\dot{\sigma}, \dot{\varepsilon}_{\mathrm{e}}\right\rangle-\left\langle\dot{\sigma}, \dot{\varepsilon}_{\mathrm{p}}\right\rangle-\left\langle\dot{\chi}, \dot{\alpha}_{\mathrm{e}}\right\rangle-\left\langle\dot{\chi}, \dot{\alpha}_{\mathrm{p}}\right\rangle \\
& \quad+\Phi^{\mathrm{t}}\left(\varepsilon_{\mathrm{e}}, \dot{\varepsilon}_{\mathrm{e}}\right)+\Pi^{\mathrm{t}}\left(\alpha_{\mathrm{e}}, \dot{\alpha}_{\mathrm{e}}\right)+\int_{\mathrm{B}} \operatorname{supp} T_{\mathrm{K}}^{(\sigma, \chi)}\left(\dot{\varepsilon}_{\mathrm{p}}, \dot{\alpha}_{\mathrm{p}}\right) \mathrm{d} B
\end{aligned}
$$

where the global potentials $\Phi^{\mathrm{t}}\left(\varepsilon_{\mathrm{e}}, \dot{\varepsilon}_{\mathrm{e}}\right), \Pi^{\mathrm{t}}\left(\alpha_{\mathrm{e}}, \dot{\alpha}_{\mathrm{e}}\right)$ are defined as follows:

$$
\begin{aligned}
& \Phi^{\mathrm{t}}\left(\varepsilon_{\mathrm{e}}, \dot{\varepsilon}_{\mathrm{e}}\right)=\int_{B} \varphi^{\mathrm{t}}\left(\varepsilon_{\mathrm{e}}, \dot{\varepsilon}_{\mathrm{e}}\right) \mathrm{d} B \\
& \Pi^{\mathrm{t}}\left(\alpha_{\mathrm{e}}, \dot{\alpha}_{\mathrm{e}}\right)=\int_{B} \pi^{\mathrm{t}}\left(\alpha_{\mathrm{e}}, \dot{\alpha}_{\mathrm{e}}\right) \mathrm{d} B
\end{aligned}
$$

and the global displacement rate potential $\Gamma^{\mathrm{t}}(\dot{u})$ is:

$$
\Gamma^{\mathrm{t}}(\dot{u})=\int_{B} \dot{b} \dot{u} \mathrm{~d} B+\int_{\partial B_{\mathrm{q}}} \dot{q} \dot{u} \mathrm{~d} s-\int_{\partial B_{\mathrm{u}}} \operatorname{ind} W \mathrm{~d} s
$$

with

$$
W=\left\{\dot{u}: \dot{u}-\dot{\bar{u}}=0 \quad \text { on } \quad \partial B_{\mathrm{u}}\right\}
$$


Its dual potential is obtained through a Legendre transformation:

$$
\begin{aligned}
& \Gamma^{\prime \mathrm{t}}(\dot{f})=\inf _{\dot{u}}\left\{\langle\dot{f}, \dot{u}\rangle-\Gamma^{\mathrm{t}}(\dot{u})\right\}=\inf _{\dot{u} \in U}\left\{\int_{B} \dot{b \dot{u}} \mathrm{~d} B+\int_{\partial B_{\mathrm{q}}} \dot{q} \dot{u} \mathrm{~d} s+\int_{\partial B_{\mathrm{u}}} \dot{r} \dot{u} \mathrm{~d} s-\int_{B} \dot{b} \dot{u} \mathrm{~d} B\right. \\
& \left.\quad-\int_{\partial B_{\mathrm{q}}} \dot{q} \dot{u} \mathrm{~d} s+\int_{\partial B_{\mathrm{u}}} \text { ind } W \mathrm{~d} s\right\}=\inf _{\dot{u} \in U}\left\{\int_{\partial B_{u}} \dot{r} \dot{u} \mathrm{~d} s+\int_{\partial B_{\mathrm{u}}} \text { ind } W \mathrm{~d} s\right\}=\int_{\partial B_{\mathrm{u}}} \dot{r} \dot{\bar{u}} \mathrm{~d} s=\langle\dot{\bar{r}} \dot{\bar{u}}\rangle_{\partial B_{\mathrm{u}}} .
\end{aligned}
$$

The functional $\Omega$ is convex w.r.t. ( $\dot{\varepsilon}_{\mathrm{e}}, \dot{\varepsilon}_{\mathrm{p}}, \dot{\alpha}_{\mathrm{e}}, \dot{\alpha}_{\mathrm{p}}$ ), concave w.r.t. $\dot{f}$ and linear w.r.t. $(\dot{u}, \dot{\sigma}, \dot{\chi})$. The solution of the structural problem is characterised as:

$$
\inf _{\left(\dot{\varepsilon}_{\mathrm{e}}, \dot{\varepsilon}_{\mathrm{p}}, \dot{\alpha}_{\mathrm{e}}, \dot{\alpha}_{\mathrm{p}}\right)} \sup _{\dot{f}} \operatorname{stat} \Omega\left(\dot{u}, \dot{u}, \dot{f}, \dot{\varepsilon}_{\mathrm{e}}, \dot{\varepsilon}_{\mathrm{p}}, \dot{\alpha}_{\mathrm{e}}, \dot{\alpha}_{\mathrm{p}}, \dot{\sigma}, \dot{\chi}\right)
$$

A 3-field functional, that generalises $\mathrm{Hu}$-Washizu's principle, is obtained after an optimisation w.r.t. $(\dot{f}$, $\left.\dot{\varepsilon}_{\mathrm{p}}, \dot{\alpha}_{\mathrm{p}}\right)$. By applying definitions $(22,5)$ we obtain:

$$
\begin{aligned}
& \Omega_{\mathrm{W}}\left(\dot{u}, \dot{\sigma}, \dot{\chi}, \dot{\varepsilon}_{\mathrm{e}}, \dot{\alpha}_{\mathrm{e}}\right)=\langle C \dot{u}, \dot{\sigma}\rangle-\Gamma^{\mathrm{t}}(\dot{u})-\left\langle\dot{\sigma}, \dot{\varepsilon}_{\mathrm{e}}\right\rangle-\left\langle\dot{\chi}, \dot{\alpha}_{\mathrm{e}}\right\rangle+\Phi^{\mathrm{t}}\left(\varepsilon_{\mathrm{e}}, \dot{\varepsilon}_{\mathrm{e}}\right)+\Pi^{\mathrm{t}}\left(\alpha_{\mathrm{e}}, \dot{\alpha}_{\mathrm{e}}\right) \\
& \quad-\int_{B} \text { ind } T_{\mathrm{K}}^{(\sigma, \chi)}(\dot{\sigma}, \dot{\chi}) \mathrm{d} B
\end{aligned}
$$

convex in $\left(\dot{u}, \dot{\varepsilon}_{\mathrm{e}}, \dot{\alpha}_{\mathrm{e}}\right)$, concave in $(\dot{\sigma}, \dot{\chi})$.

A further optimisation w.r.t. the kinematic variables $\left(\dot{\varepsilon}_{\mathrm{e}}, \dot{\alpha}_{\mathrm{e}}\right)$, after applying Legendre transformations, leads to a generalised form of the Hellinger-Reissner functional for inelastic rate problems:

$$
\Omega_{\mathrm{R}}(\dot{u}, \dot{\sigma}, \dot{\chi})=\langle C \dot{u}, \dot{\sigma}\rangle-\Gamma^{\mathrm{t}}(\dot{u})-\Phi^{\prime \mathrm{t}}(\sigma, \dot{\sigma})-\Pi^{\prime \mathrm{t}}(\chi, \dot{\chi})-\int_{B} \text { ind } T_{\mathrm{K}}^{(\sigma, \chi)}(\dot{\sigma}, \dot{\chi}) \mathrm{d} B
$$

where $\Phi^{\prime \mathrm{t}}(\sigma, \dot{\sigma}) \mathrm{e} \Pi^{\prime \mathrm{t}}(\chi, \dot{\chi})$ are conjugate potentials. $\Omega_{\mathrm{R}}$ is concave in $(\dot{\sigma}, \dot{\chi})$ and convex in $\dot{u}$.

The generalised forms of the Prager-Hodge and Greenberg functionals are obtained after optimisation with respect to $\dot{u}$ or $(\dot{\sigma}, \dot{\chi})$. Since

$$
\sup _{\dot{u}}\left\{\langle C \dot{u}, \dot{\sigma}\rangle-\Gamma^{\mathrm{t}}(\dot{u})\right\}=\sup _{\dot{u}}\left\{\langle C \dot{u}, \dot{\sigma}\rangle-\langle\dot{f}, \dot{u}\rangle+\langle\dot{f}, \dot{u}\rangle-\Gamma^{\mathrm{t}}(\dot{u})\right\}=\Gamma^{\prime \mathrm{t}}(\dot{f}(\dot{\sigma}))
$$

subject to

$$
\langle C \dot{u}, \dot{\sigma}\rangle-\langle\dot{f}, \dot{u}\rangle=0
$$

the Prager-Hodge functional becomes:

$$
\Omega_{\mathrm{c}}(\dot{\sigma}, \dot{\chi})=-\Phi^{\prime \mathrm{t}}(\sigma, \dot{\sigma})-\Pi^{\prime \mathrm{t}}(\chi, \dot{\chi})+\langle\dot{r}, \dot{\bar{u}}\rangle-\int_{B} \text { ind } T_{\mathrm{K}}^{(\sigma, \chi)}(\dot{\sigma}, \dot{\chi}) \mathrm{d} B
$$

subject to equilibrium conditions

$$
\left\langle C^{\prime} \dot{\sigma}, \tilde{\dot{u}}\right\rangle=\langle\dot{f}, \tilde{\dot{u}}\rangle \quad \forall \tilde{\dot{u}} \in U \quad \text { in } \quad B \cup \partial B_{\mathrm{q}} .
$$

The relevant structural problem in terms of rates of stress and thermodynamic force can be formulated as follows: 


$$
\sup _{(\dot{\sigma}, \dot{\chi}) \in Q} \Omega_{\mathrm{c}}(\dot{\sigma}, \dot{\chi}) \quad Q=\left\{(\dot{\sigma}, \dot{\chi}):\left\langle C^{\prime} \dot{\sigma}, \tilde{\dot{u}}\right\rangle=\langle\dot{f}, \tilde{\dot{u}}\rangle \quad \forall \tilde{\dot{u}} \in U\right\}
$$

For the Greenberg functional the following form is obtained:

$$
\Omega_{\mathrm{u}}(\dot{u})=\Phi^{\mathrm{ep}}(C \dot{u})-\Gamma^{\mathrm{t}}(\dot{u})
$$

where

$$
\Phi^{\mathrm{ep}}(\dot{u})=\sup _{(\dot{\sigma}, \dot{\gamma})}\left[\langle C \dot{u}, \dot{\sigma}\rangle-\Phi^{\prime \mathrm{t}}(\sigma, \dot{\sigma})-\Pi^{\prime \mathrm{t}}(\chi, \dot{\chi})-\int_{B} \text { ind } T_{\mathrm{K}}^{(\sigma, \chi)}(\dot{\sigma}, \dot{\chi}) \mathrm{d} B\right] .
$$

This functional, convex in $\dot{u}$, is the starting point for classical displacement methods. It can be proved that the optimality condition of (24) leads to the generalised return mapping algorithm.

\subsection{Formulations involving actual values of the variables}

The variational principles of the previous section can be reformulated in terms of actual values of the variables, rather than their rates. This formulation allows the total compatibility and equilibrium conditions to be satisfied in the final state, reducing in principle the approximation errors. However, it is necessary to approximate the flow rule that involves deformation rates.

Introducing a fully implicit integration scheme for the kinematic variables, one has

$$
\begin{aligned}
& \Delta \varepsilon_{\mathrm{p}}=\varepsilon_{\mathrm{p}}(t+\Delta t)-\varepsilon_{\mathrm{p}_{0}}=\dot{\varepsilon}_{\mathrm{p}}(t+\Delta t) \Delta t \\
& \Delta \alpha_{\mathrm{p}}=\alpha_{\mathrm{p}}(t+\Delta t)-\alpha_{\mathrm{p}_{0}}=\dot{\alpha}_{\mathrm{p}}(t+\Delta t) \Delta t
\end{aligned}
$$

$\varepsilon_{\mathrm{p}_{0}}$ and $\alpha_{\mathrm{p}_{0}}$ being their values at the end of the previous step. The finite increment form of the constitutive relations is:

$$
\left(\frac{\Delta \varepsilon_{\mathrm{p}}}{\Delta t}, \frac{\Delta \alpha_{\mathrm{p}}}{\Delta t}\right) \in \partial \text { ind } K(\sigma(t), \chi(t))=N_{\mathrm{K}}(\sigma(t), \chi(t)) .
$$

Since $N_{\mathrm{K}}$ is a concave cone and $\Delta \mathrm{t} \geq 0(25)$ can be reformulated as:

$$
\left(\Delta \varepsilon_{p}, \Delta \alpha_{\mathrm{p}}\right) \in \partial \text { ind } K(\sigma(t), \chi(t))=N_{\mathrm{K}}(\sigma(t), \chi(t))
$$

and the finite step problem is ruled by the functional:

$$
\begin{aligned}
& \Omega\left(u, f, \varepsilon_{\mathrm{e}}, \varepsilon_{\mathrm{p}}, \alpha_{\mathrm{e}}, \alpha_{\mathrm{p}}, \sigma, \chi\right)=\langle C u, \sigma\rangle+\Gamma^{\prime}(f)-\langle f, u\rangle-\left\langle\sigma, \varepsilon_{\mathrm{e}}\right\rangle-\left\langle\sigma, \varepsilon_{\mathrm{p}}\right\rangle+\left\langle\sigma, \varepsilon_{\mathrm{p}_{0}}\right\rangle+-\left\langle\chi, \alpha_{\mathrm{e}}\right\rangle \\
& \quad-\left\langle\chi, \alpha_{\mathrm{p}}\right\rangle+\left\langle\chi, \alpha_{\mathrm{p}_{0}}\right\rangle+\Phi_{\mathrm{e}}\left(\varepsilon_{\mathrm{e}}, \varepsilon_{\mathrm{e}}\right)+\Pi\left(\alpha_{\mathrm{e}}, \alpha_{\mathrm{e}}\right)+\operatorname{supp} K\left(\varepsilon_{\mathrm{p}}-\varepsilon_{\mathrm{p}_{0}}, \alpha_{\mathrm{p}}-\alpha_{\mathrm{p}_{0}}\right) .
\end{aligned}
$$

The whole family of variational functionals described in section 3.1 can be consistently derived. For instance, the generalised finite increment forms of the Hellinger-Reissner and Prager-Hodge functionals are:

$$
\begin{aligned}
& \Omega_{\mathrm{R}}(u, \sigma, \chi)=\langle C u, \sigma\rangle+\left\langle\sigma, \varepsilon_{\mathrm{p}_{0}}\right\rangle+\left\langle\chi, \alpha_{\mathrm{p}_{0}}\right\rangle-\Gamma(u)-\Phi^{\prime}(\sigma)-\Pi^{\prime}(\chi)-\int_{B} \text { ind } K(\sigma, \chi) \mathrm{d} B \\
& \Omega_{\mathrm{c}}(\sigma, \chi)=\left\langle\sigma, \varepsilon_{\mathrm{p}_{0}}\right\rangle+\left\langle\chi, \alpha_{\mathrm{p}_{0}}\right\rangle-\Phi^{\prime \mathrm{t}}(\sigma)-\Pi^{\prime}(\chi)+\Gamma^{\prime}(f)-\int_{B} \text { ind } K(\sigma, \chi) \mathrm{d} B
\end{aligned}
$$


subject to equilibrium conditions

$$
\left\langle C^{\prime} \sigma, \tilde{u}\right\rangle=\langle f, \tilde{u}\rangle \quad \forall \tilde{u} \in U \quad \text { in } \quad B \cup \partial B_{\mathrm{q}} .
$$

\subsection{Incremental formulations}

A finite increment formulation will now be derived from the rate form of the Hellinger-Reissner functional in section 3.1, which will be the starting point for the numerical algorithm illustrated in section 4.

The solution of the elastoplastic problem can be achieved if a subdivision of the load history into $n$ finite increments, corresponding to the instants $t_{0}, t_{1}, \ldots, t_{\mathrm{n}}$, is introduced. All variable rates are substituted with finite increments in the step. At the time $t+\Delta t$ the values of $(\sigma, \chi)$ are given by:

$$
\sigma(t+\Delta t)=\sigma(t)+\int_{t}^{t+\Delta t} \dot{\sigma}(t) \mathrm{d} t ; \quad \chi(t+\Delta t)=\chi(t)+\int_{t}^{t+\Delta t} \dot{\chi}(t) \mathrm{d} t .
$$

A similar formula can be introduced for the displacement $u$. For each variable the following integration rule can be used:

$$
\sigma(t+\Delta t)=\sigma(t)+\left[(1-\beta) \dot{\sigma}(t)_{+}+\beta \dot{\sigma}(t+\Delta t)\right] \Delta t \quad 0 \leq \beta \leq 1 .
$$

If $\beta=1$ the fully implicit integration scheme is obtained (note that in (26) the left derivative appears):

$$
\sigma(t+\Delta t)=\sigma(t)+\dot{\sigma}(t+\Delta t) \Delta t=\sigma(t)+\Delta \sigma(t+\Delta t) .
$$

This method ensures that the compatibility conditions are satisfied at the end of the step and it will be used in what follows.

The increment of plastic deformations is given by:

$$
\left[\begin{array}{c}
\Delta \varepsilon_{\mathrm{p}} \\
\Delta \alpha_{\mathrm{p}}
\end{array}\right]=\int_{t}^{t+\Delta t}\left[\begin{array}{c}
\dot{\varepsilon}_{\mathrm{p}} \\
\dot{\alpha}_{\mathrm{p}}
\end{array}\right] \mathrm{d} t
$$

The plastic rates are evaluated using the flow rule (11) that contains the right derivative of the stresses, which is not known at time $t+\Delta t$ (see Eq. (26)).

Therefore a relaxed form of the flow rule is adopted, employing inclusion (12):

$$
\left[\begin{array}{c}
\Delta \varepsilon_{\mathrm{p}} \\
\Delta \alpha_{\mathrm{p}}
\end{array}\right] \in \int_{t}^{t+\Delta t} \partial \text { ind } T_{\mathrm{K}}^{(\sigma, \chi)}\left(\dot{\sigma}_{+}, \dot{\chi}_{+}\right) \mathrm{d} t \subseteq \int_{t}^{t+\Delta t} N_{\mathrm{K}}(\sigma, \chi) \mathrm{d} t=\int_{t}^{t+\Delta t} \lambda \partial g(\sigma, \chi) \mathrm{d} t .
$$

The integration has to be carried out along an admissible path. Hypothesis (26) requires that this integration be performed along a secant path from the point at time $t$ to the point at time $t+\Delta t$. From the convexity of $K$ it follows that the stress path is non external to $K$ and the integral in (27) is non zero only at the end of the interval, except for the special case of a flat boundary (see Fig. 4).

The result is therefore:

$$
\left[\begin{array}{l}
\Delta \varepsilon_{\mathrm{p}} \\
\Delta \alpha_{\mathrm{p}}
\end{array}\right] \in \lambda \partial g(\sigma+\Delta \sigma, \chi+\Delta \chi) \text {. }
$$

Formula (28) is equivalent to stating that 


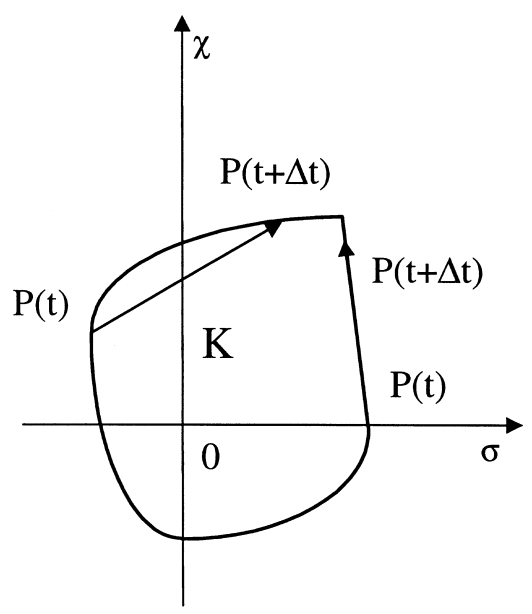

Fig. 4. Admissible paths in a finite step.

$$
\left[\begin{array}{c}
\Delta \varepsilon_{\mathrm{p}} \\
\Delta \alpha_{\mathrm{p}}
\end{array}\right]=\left[\begin{array}{c}
\dot{\varepsilon}_{\mathrm{p}}(t+\Delta t) \\
\dot{\alpha}_{\mathrm{p}}(t+\Delta t)
\end{array}\right] \Delta t
$$

which is in agreement with the implicit scheme adopted. Note that in (29) the scalar factor $\Delta t$ is immaterial, since $N_{\mathrm{K}}$ is a cone.

In conclusion the incremental form of the functionals $\Omega_{\mathrm{R}}$ and $\Omega_{\mathrm{c}}$ is:

$$
\begin{aligned}
& \Omega_{\mathrm{R}}(\Delta u, \Delta \sigma, \Delta \chi)=\langle C \Delta u, \Delta \sigma\rangle-\Gamma^{\prime \mathrm{t}}(\Delta u)-\Phi^{\prime \mathrm{t}}(\Delta \sigma)-\Pi^{\prime \mathrm{t}}(\Delta \chi)-\int_{B} \text { ind } K(\sigma+\Delta \sigma, \chi+\Delta \chi) \mathrm{d} B \\
& \Omega_{\mathrm{c}}(\Delta \sigma, \Delta \chi)=-\Phi^{\prime \mathrm{t}}(\Delta \sigma)-\Pi^{\prime \mathrm{t}}(\Delta \chi)+\langle\Delta r, \Delta \bar{u}\rangle-\int_{B} \text { ind } K(\sigma+\Delta \sigma, \chi+\Delta \chi) \mathrm{d} B
\end{aligned}
$$

subject to

$$
\left\langle C^{\prime} \Delta \sigma-\Delta f, \Delta u\right\rangle=0
$$

where the tangent potentials are evaluated as:

$$
\begin{aligned}
& \Phi^{\prime \mathrm{t}}(\Delta \sigma)=\frac{1}{2}\left\langle\nabla^{2} \Phi^{\mathrm{t}}(\sigma(t+\Delta t)) \Delta \sigma, \Delta \sigma\right\rangle=\frac{1}{2}\left\langle E_{\mathrm{t}}(\sigma(t+\Delta t)) \Delta \sigma, \Delta \sigma\right\rangle \\
& \Pi^{\prime \mathrm{t}}(\Delta \chi)=\frac{1}{2}\left\langle\nabla^{2} \Pi^{\mathrm{t}}(\chi(t+\Delta t)) \Delta \chi, \Delta \chi\right\rangle=\frac{1}{2}\left\langle H_{\mathrm{t}}(\chi(t+\Delta t)) \Delta \chi, \Delta \chi\right\rangle .
\end{aligned}
$$

\subsection{Regularisation}

In section 2 it was pointed out that the constitutive equations take the form of perfect unilateral conditions. For these classes of problems Augmented Lagrangian Regularisation (A.L.R.) has been 
successfully introduced and it has several computational and analytical advantages (see, e.g. Glowinski and Le Tallec, 1989).

First, by using an A.L. formulation one has a faster convergence rate than by using normal Lagrangian functionals (Cuomo et al., 1997). More importantly, A.L.R. becomes especially effective when the elastic deformations are much smaller than the inelastic ones, so they do not introduce enough smoothing on the problem, or when non-convex functionals are involved, as could be the case with softening. Below it will be shown that the use of A.L.R. leads to effective computational schemes that could achieve a substantial reduction in computational effort w.r.t. the usual Lagrangian methods.

The functional (31) is made differentiable through the Augmented Lagrangian Regularisation:

$$
\text { ind } K(\sigma+\Delta \sigma, \chi+\Delta \chi)=\sup _{\lambda \geq 0}\left\{\frac{1}{2} \mu g^{2}+\lambda g\right\}=\sup _{\lambda}\left\{\frac{1}{2} \mu \bar{g}^{2}+\lambda \bar{g}\right\}
$$

$\lambda$ being the Lagrangian plastic multiplier, $\mu$ a positive constant penalty parameter and

$$
\bar{g}=\max \left(g,-\frac{\lambda}{\mu}\right)
$$

The function $\bar{g}$ converts the inequality constraint $g(\sigma+\Delta \sigma, \chi+\Delta \chi) \leq 0$ into an equality one $\bar{g}(\sigma+\Delta \sigma$, $\chi+\Delta \chi)=0$, removing the sign restriction on the Lagrangian multiplier and preserving the functional continuity in the neighbourhood of the solution (Bertsekas, 1982).

Problem (23) thus turns into a saddle point problem:

$$
\begin{aligned}
& \sup _{(\Delta \sigma, \Delta \chi) \in \hat{Q}} \inf _{\lambda} \Omega_{\mathrm{c}}^{\mathrm{AL}}(\Delta \sigma, \Delta \chi, \lambda) \\
& \hat{Q}=\left\{(\Delta \sigma, \Delta \chi): C^{\prime} \Delta \sigma=\Delta f\right\}
\end{aligned}
$$

where $\Omega_{\mathrm{c}}^{\mathrm{AL}}(\Delta \sigma, \Delta \chi, \lambda)$ is the following differentiable functional:

$$
\begin{gathered}
\Omega_{\mathrm{c}}^{\mathrm{AL}}(\Delta \sigma, \Delta \chi, \lambda)=-\frac{1}{2}\left\langle E_{\mathrm{t}}{ }^{1} \Delta \sigma, \Delta \sigma\right\rangle-\frac{1}{2}\left\langle H_{\mathrm{t}}{ }^{1} \Delta \chi, \Delta \chi\right\rangle+\langle\Delta r(\Delta \sigma), \Delta \bar{u}\rangle \\
-\int_{B}\left\{\frac{1}{2} \mu \bar{g}^{2}(\sigma+\Delta \sigma, \chi+\Delta \chi)+\lambda \bar{g}(\sigma+\Delta \sigma, \chi+\Delta \chi)\right\} \mathrm{d} B .
\end{gathered}
$$

According to (28) the increments of the plastic deformations and internal variables are given by the following expressions:

$$
\begin{aligned}
& \Delta \varepsilon_{\mathrm{p}}=\nabla_{\sigma}\left(\frac{1}{2} \mu \bar{g}^{2}+\lambda^{*} \bar{g}\right)=\left(\mu g+\lambda^{*}\right) \nabla_{\sigma} g=\lambda^{*} \nabla_{\sigma} g \\
& \Delta \alpha_{\mathrm{p}}=\nabla_{\chi}\left(\frac{1}{2} \mu \bar{g}^{2}+\lambda^{*} \bar{g}\right)=\left(\mu g+\lambda^{*}\right) \nabla_{\chi} g=\lambda^{*} \nabla_{\chi} g
\end{aligned}
$$

where $\lambda^{*}$ is the value of the plastic multiplier at solution and the terms $\mu g \nabla_{\sigma} g$ and $\mu g \nabla_{\chi} g$ vanish since the constraint is satisfied $(\bar{g}=g=0)$. 


\section{Discrete structural problem}

\subsection{Discretised variational formulation}

A discretisation into $n_{\mathrm{e}}$ finite elements $B_{\mathrm{e}}$ is introduced. The increments of the stresses, thermodynamic forces and displacements are thus given by

$$
\Delta \sigma=\mathbf{N}_{\sigma} \Delta \mathbf{s}, \quad \Delta \chi=\mathbf{N}_{\chi} \Delta \mathbf{c}, \quad \Delta u=\mathbf{N}_{\mathrm{u}} \Delta \mathbf{d}, \quad \Delta \bar{u}=\mathbf{N}_{\mathrm{u}} \Delta \mathbf{d}
$$

$\mathbf{N}_{\sigma}, \mathbf{N}_{\chi}, \mathbf{N}_{\mathrm{u}}, \mathbf{N}_{\overline{\mathrm{u}}}$ being suitable shape function matrices.

It is noticed that a necessary condition for the existence of a solution to the equilibrium equations is that the dimension of the nodal stress space be greater than that of the nodal displacement space (Zienkievicz and Taylor, 1989).

The discretised form of the Hellinger-Reissner functional, given by Eq. (30), is:

$$
\begin{aligned}
& \Omega_{\mathrm{R}}(\Delta \mathbf{d}, \Delta \mathbf{d}, \Delta \mathbf{s}, \Delta \mathbf{c})=\int_{B} C \mathbf{N}_{\mathrm{u}} \Delta \mathbf{d} \cdot \mathbf{N}_{\sigma} \Delta \mathbf{s} \mathrm{d} B+\int_{\partial B_{\mathrm{u}}} C \mathbf{N}_{\mathrm{u}} \Delta \mathbf{d} \cdot \mathbf{N}_{\sigma} \Delta \mathbf{s} \mathrm{d} s-\frac{1}{2} \int_{B} E_{\mathrm{t}}{ }^{1} \mathbf{N}_{\sigma} \Delta \mathbf{s} \cdot \mathbf{N}_{\sigma} \Delta \mathbf{s} \mathrm{d} B \\
& \quad-\int_{B} \Delta b \mathbf{N}_{\mathrm{u}} \Delta \mathbf{d} \mathrm{d} B+-\int_{\partial B_{\mathrm{q}}} \Delta q \mathbf{N}_{\mathrm{u}} \Delta \mathbf{d} \mathrm{d} s-\int_{\partial B_{\mathrm{u}}} \Delta r \mathbf{N}_{\mathrm{u}} \Delta \mathbf{d} \mathrm{d} s+-\int_{B} H_{\mathrm{t}}{ }^{1} \mathbf{N}_{\chi} \Delta \mathbf{c} \cdot \mathbf{N}_{\chi} \Delta \mathbf{c} \mathrm{d} B \\
& \quad-\int_{B} \frac{1}{2} \mu \bar{g}^{2}(\mathbf{s}+\Delta \mathbf{s}, \mathbf{c}+\Delta \mathbf{c})+\lambda \bar{g}(\mathbf{s}+\Delta \mathbf{s}, \mathbf{c}+\Delta \mathbf{c}) \mathrm{d} B
\end{aligned}
$$

where $\Delta \mathbf{d}$ are the displacements of the nodes belonging to the constrained part of the boundary.

The variation of this functional w.r.t. $\Delta \mathbf{d}$ and $\Delta \overline{\mathbf{d}}$ gives the discretised equilibrium equations:

$$
\begin{aligned}
& \mathbf{C} \Delta \mathbf{s}=\Delta \mathbf{b}+\Delta \mathbf{q}=\Delta \mathbf{p} \quad \text { in } \quad B \cup \partial B_{\mathrm{q}} \\
& \mathbf{Q} \Delta \mathbf{s}=\Delta \mathbf{r} \quad \text { on } \quad \partial B_{\mathrm{u}}
\end{aligned}
$$

The equilibrium operators $\mathbf{C}$ and $\mathbf{Q}$ are defined as:

$$
\mathbf{C}=\int_{B}\left(C \mathbf{N}_{\mathrm{u}}\right)^{\mathrm{T}} \mathbf{N}_{\sigma} \mathrm{d} B ; \quad \mathbf{Q}=\int_{\partial B_{\mathrm{u}}}\left(C \mathbf{N}_{\mathrm{u}}\right)^{\mathrm{T}} \mathbf{N}_{\sigma} \mathrm{d} s
$$

and $\Delta \mathbf{p}$ and $\Delta \mathbf{r}$ are the nodal vectors of the external forces and the reactions, defined as:

$$
\begin{aligned}
& \Delta \mathbf{p}=\Delta \mathbf{b}+\Delta \mathbf{q}=\int_{B} \mathbf{N}_{\mathrm{u}}^{\mathrm{T}} \Delta b \mathrm{~d} B+\int_{\partial B_{\mathrm{q}}} \mathbf{N}_{\mathrm{u}}^{\mathrm{T}} \Delta q \mathrm{~d} s \\
& \Delta \mathbf{r}=\int_{\partial B_{\mathrm{u}}} \mathbf{N}_{\mathrm{u}}^{\mathrm{T}} \Delta r \mathrm{~d} s .
\end{aligned}
$$

The discretised form of the functional $\Omega_{\mathrm{c}}(\Delta \sigma, \Delta \chi)$, given by Eq. (31), is: 


$$
\begin{aligned}
& \Omega_{\mathrm{c}}(\Delta \mathbf{s}, \Delta \mathbf{c})=-\frac{1}{2} \int_{B} \mathbf{N}_{\sigma}^{\mathrm{T}} E_{\mathrm{t}}{ }^{1} \mathbf{N}_{\sigma} \Delta \mathbf{s} \Delta \mathbf{s} \mathrm{d} B-\frac{1}{2} \int_{B} \mathbf{N}_{\chi}^{\mathrm{T}} H_{\mathrm{t}}{ }^{1} \mathbf{N}_{\chi} \Delta \mathbf{c} \Delta \mathbf{c} \mathrm{d} B+\int_{\partial B_{\mathrm{u}}} \Delta r \mathbf{N}_{\mathrm{u}} \Delta \mathbf{d} \mathrm{d} s \\
& \quad-\int_{B} \text { ind } K(\mathbf{s}+\Delta \mathbf{s}, \mathbf{c}+\Delta \mathbf{c}) \mathrm{d} B
\end{aligned}
$$

subject to the equilibrium conditions in the inside and on the boundary.

Introducing the tangent flexibility and hardening matrices

$$
\mathbf{F}=\int_{B} \mathbf{N}_{\sigma}^{\mathrm{T}} E_{\mathrm{t}}{ }^{1} \mathbf{N}_{\sigma} \mathrm{d} B ; \quad \mathbf{G}=\int_{B} \mathbf{N}_{\chi}^{\mathrm{T}} H_{\mathrm{t}}{ }^{1} \mathbf{N}_{\chi} \mathrm{d} B
$$

the functional (34) is written in compact form:

$$
\begin{aligned}
& \Omega_{\mathrm{c}}(\Delta \mathbf{s}, \Delta \mathbf{c})=-\frac{1}{2} \mathbf{F} \Delta \mathbf{s} \Delta \mathbf{s}-\frac{1}{2} \mathbf{G} \Delta \mathbf{c} \Delta \mathbf{c}+\mathbf{Q} \Delta \mathbf{s} \Delta \mathbf{d}-\int_{B} \text { ind } K(\mathbf{s}+\Delta \mathbf{s}, \mathbf{c}+\Delta \mathbf{c}) \mathrm{d} B \\
& \Delta \mathbf{s} \in Y \quad Y=\left\{\Delta \mathbf{s}: \mathbf{C} \Delta \mathbf{s}=\Delta \mathbf{p} \quad \text { in } \quad B \cup \partial B_{\mathrm{q}}, \quad \mathbf{Q} \Delta \mathbf{s}=\Delta \mathbf{r} \quad \text { on } \quad \partial B_{\mathrm{u}}\right\}
\end{aligned}
$$

$Y$ being the set of nodal stresses satisfying the equilibrium equations in the weak sense.

The equilibrium constraint for the functional $\Omega_{\mathrm{c}}$ can be removed through a reduction of the variables $\Delta \mathbf{s}$ to the self-equilibrated stresses $\Delta \mathbf{s}_{0}$. By re-arranging the columns of the matrix $\mathbf{C}$, the following partition can be obtained:

$$
\mathbf{C}=\left[\begin{array}{ll}
\mathbf{C}_{0} & \mathbf{C}_{1}
\end{array}\right]
$$

where $\mathbf{C}_{1}$ is square and non singular. If the same decomposition is carried out on $\Delta \mathbf{s}$, the equilibrium equations in $B \cup \partial B_{\mathrm{q}}$ can be written as

$$
\left[\begin{array}{ll}
\mathbf{C}_{0} & \mathbf{C}_{1}
\end{array}\right]\left[\begin{array}{c}
\Delta \mathbf{s}_{0} \\
\Delta \mathbf{s}_{1}
\end{array}\right]=\Delta \mathbf{p}
$$

where $\Delta \mathbf{s}_{1}$ is a vector of nodal stress increments equilibrating the external load increments.

As $\mathbf{C}_{1}$ is non singular, the following expression for $\Delta \mathbf{s}_{1}$ is obtained:

$$
\Delta \mathbf{s}_{1}=-\mathbf{C}_{1}{ }^{1} \mathbf{C}_{0} \Delta \mathbf{s}_{0}+\mathbf{C}_{1}{ }^{1} \Delta \mathbf{p}=-\tilde{\mathbf{C}}_{0} \Delta \mathbf{s}_{0}+\Delta \tilde{\mathbf{p}}
$$

In the numerical implementation $\tilde{\mathbf{C}}_{0}$ and $\Delta \tilde{\mathbf{p}}$ are obtained directly by means of a Gauss reduction of the matrix $\mathbf{C}$ and of the vector $\Delta \mathbf{p}$ such that $\mathbf{C}_{1} \equiv \mathbf{I}$.

Introducing the reduced stress increments in (35)

$$
\Delta \mathbf{s}=\left[\begin{array}{l}
\Delta \mathbf{s}_{0} \\
\Delta \mathbf{s}_{1}
\end{array}\right]=\left[\begin{array}{l}
\mathbf{I} \\
-\tilde{\mathbf{C}}_{0}
\end{array}\right] \Delta \mathbf{s}_{0}+\left[\begin{array}{l}
\mathbf{0} \\
\Delta \tilde{\mathbf{p}}
\end{array}\right]=\mathbf{R} \Delta \mathbf{s}_{0}+\Delta \mathbf{v}
$$

the functional $\Omega_{\mathrm{c}}(\Delta \mathbf{s}, \Delta \mathbf{c})$ becomes:

$$
\begin{aligned}
& \Omega_{\mathrm{c}}\left(\Delta \mathbf{s}_{0}, \Delta \mathbf{c}\right)=-\frac{1}{2} \mathbf{R}^{\mathrm{T}} \mathbf{F} \mathbf{R} \Delta \mathbf{s}_{0} \Delta \mathbf{s}_{o}-\frac{1}{2} \mathbf{G} \Delta \mathbf{c} \Delta \mathbf{c}-\mathbf{R}^{\mathrm{T}} \mathbf{F} \Delta \mathbf{v} \Delta \mathbf{s}_{0}+\mathbf{R}^{\mathrm{T}} \mathbf{Q}^{\mathrm{t}} \Delta \mathbf{d} \Delta \mathbf{s}_{0}+-\frac{1}{2} \mathbf{F} \Delta \mathbf{v} \Delta \mathbf{v}+\mathbf{Q} \Delta \mathbf{v} \Delta \mathbf{d} \\
& \quad-\int_{B} \text { ind } K\left(\mathbf{s}_{0}+\Delta \mathbf{s}_{0}, \mathbf{c}+\Delta \mathbf{c}\right) \mathrm{d} B .
\end{aligned}
$$

The indicator function in formula (37) is regularised according to the Augmented Lagrangian scheme of 
Eq. (32), that needs to be better specified. It derives from the duality pairing $\langle\mu \bar{g}+\lambda, \bar{g}\rangle$ where, elementwise, $\bar{g} \in \mathscr{C}_{0}^{0}, \lambda \in \mathscr{C}^{\prime}$. Therefore the multipliers $\lambda$ are measures and the integral in (37) has to be understood in the distribution sense. It is numerically evaluated assuming a Dirac distribution for the multipliers with singularity at prescribed points, which play the role of check points for the plasticity constraints:

$$
\langle\mu \bar{g}+\lambda, \bar{g}\rangle=\sum_{i=1}^{n_{\mathrm{e}}} \sum_{j=1}^{n_{\mathrm{cpe}}}\left[\frac{1}{2} \mu \bar{g}^{2}\left(\mathbf{s}_{0}+\Delta \mathbf{s}_{0}, \mathbf{c}+\Delta \mathbf{c}\right)+\lambda \bar{g}\left(\mathbf{s}_{0}+\Delta \mathbf{s}_{0}, \mathbf{c}+\Delta \mathbf{c}\right)\right]_{j}^{i}
$$

where the sum is made on the $n_{\text {cpe }}$ check points of the element. Note the absence in (38) of the Jacobian term.

The Gauss points of the elements were used as check points. The choice of the check points affects the convergence of the discretised solution, but this aspect is not addressed in the present paper.

Therefore, the functional $\Omega_{\mathrm{c}}\left(\Delta \mathbf{s}_{0}, \Delta \mathbf{c}\right)$, eliminating unessential constant terms and after a sign reversal, becomes:

$$
\begin{aligned}
& \Omega_{\mathrm{c}}^{\mathrm{AL}}\left(\Delta \mathbf{s}_{0}, \Delta \mathbf{c}, \lambda\right)=\frac{1}{2} \mathbf{R}^{\mathrm{T}} \mathbf{F} \mathbf{R} \Delta \mathbf{s}_{0} \Delta \mathbf{s}_{0}+\frac{1}{2} \mathbf{G} \Delta \mathbf{c} \Delta \mathbf{c}+\mathbf{R}^{\mathrm{T}} \mathbf{F} \Delta \mathbf{v} \Delta \mathbf{s}_{0}--\mathbf{R}^{\mathrm{T}} \mathbf{Q}^{\mathrm{T}} \Delta \mathbf{d} \Delta \mathbf{s}_{0} \\
& +\sum_{j=1}^{n_{\mathrm{cp}}}\left[\frac{1}{2} \mu \bar{g}^{2}\left(\mathbf{s}_{0}+\Delta \mathbf{s}_{0}, \mathbf{c}+\Delta \mathbf{c}\right)+\lambda \bar{g}\left(\mathbf{s}_{0}+\Delta \mathbf{s}_{0}, \mathbf{c}+\Delta \mathbf{c}\right)\right]_{j}
\end{aligned}
$$

$n_{\mathrm{cp}}$ being the total number of check points in $B$. The solution of the structural problem is given by:

$$
\min _{\left(\Delta \mathbf{s}_{0}, \Delta \mathbf{c}\right)} \max _{\lambda} \Omega_{\mathrm{c}}^{\mathrm{AL}}\left(\Delta \mathbf{s}_{0}, \Delta \mathbf{c}, \lambda\right)
$$

\subsection{Displacement calculation}

The variation w.r.t. $\Delta \mathbf{S}$ of the discretised form of the Hellinger-Reissner functional (33) yields the kinematic compatibility equation:

$$
\mathbf{C}^{\mathrm{T}} \Delta \mathbf{d}=\mathbf{F} \Delta \mathbf{s}-\mathbf{Q}^{\mathrm{T}} \Delta \mathbf{d}+\sum_{j=1}^{n_{\mathrm{cp}}}\left[\mathbf{N}_{\sigma}^{\mathrm{T}}\left(\mu \bar{g}+\Delta \lambda^{*}\right) \nabla_{\sigma} \bar{g}\right]_{j}
$$

which means that the total deformation vector $\mathbf{C}^{\mathrm{T}} \Delta \mathbf{d}$ is obtained as the sum of the elastic and plastic parts minus $\mathbf{Q}^{\mathrm{T}} \Delta \overline{\mathbf{d}}$, i.e. the deformations due to the imposed boundary displacements.

Defining the deformation vectors as:

$$
\Delta \mathbf{e}=\Delta \mathbf{e}_{\mathrm{e}}+\Delta \mathbf{e}_{\mathrm{p}}, \quad \Delta \mathbf{e}_{\mathrm{e}}=\mathbf{F} \Delta \mathbf{s}, \quad \Delta \mathbf{e}_{\mathrm{p}}=\sum_{j=1}^{n_{\mathrm{cp}}}\left[\mathbf{N}_{\sigma}^{\mathrm{T}}\left(\mu \bar{g}+\lambda^{*}\right) \nabla_{\sigma} \bar{g}\right]_{j}
$$

and introducing the decomposition of matrix $\mathbf{C}$ shown in 4.1, relation (41) can be written as:

$$
\left[\begin{array}{l}
\tilde{\mathbf{C}}_{0}^{\mathrm{T}} \\
\mathbf{I}
\end{array}\right] \Delta \mathbf{d}=\left[\begin{array}{l}
\Delta \mathbf{e}_{0} \\
\Delta \mathbf{e}_{1}
\end{array}\right]-\left[\begin{array}{l}
\mathbf{Q}_{0}^{\mathrm{T}} \\
\mathbf{Q}_{1}^{\mathrm{T}}
\end{array}\right] \Delta \mathbf{d}
$$

where 


$$
\left[\begin{array}{c}
\Delta \mathbf{e}_{0} \\
\Delta \mathbf{e}_{1}
\end{array}\right]=\left[\begin{array}{cc}
\mathbf{F}_{00} & \mathbf{F}_{01} \\
\mathbf{F}_{10} & \mathbf{F}_{11}
\end{array}\right]\left[\begin{array}{c}
\Delta \mathbf{s}_{0} \\
\Delta \mathbf{s}_{1}
\end{array}\right]+\sum_{j=1}^{n_{\mathrm{cp}}}\left[\begin{array}{c}
\mathbf{N}_{\sigma_{0}}^{\mathrm{T}} \\
\mathbf{N}_{\sigma_{1}}^{\mathrm{T}}
\end{array}\right]\left(\mu \bar{g}+\lambda^{*}\right) \nabla_{\sigma} \bar{g}
$$

It should be pointed out that $\mathbf{Q}_{0}$ and $\mathbf{Q}_{1}$ are the matrices that derive from rearrangement of the columns of the boundary equilibrium operator $\mathbf{Q}$. In fact the Gauss reduction performed on the rows of $\mathbf{C}$ to obtain the partition $\mathbf{C}=\left[\begin{array}{ll}\tilde{\mathbf{C}}_{0} & \mathbf{I}\end{array}\right]$ does not apply on $\mathbf{Q}$ or $\Delta \mathbf{r}$.

The second equation of (42) allows the displacements of the unconstrained nodes to be evaluated in the form:

$$
\Delta \mathbf{d}=\Delta \mathbf{e}_{1}-\mathbf{Q}_{1}^{\mathrm{t}} \Delta \mathbf{d}
$$

The first equation in (42) is an identity since it is the Euler-Lagrange equation of the functional $\Omega_{\mathrm{c}}^{\mathrm{AL}}\left(\Delta \mathbf{s}_{0}, \Delta \mathbf{c}, \lambda\right)$ (Eq. (39)). Indeed, by substituting $\Delta \mathbf{d}$ as given by (44) into the first equation in (42) one has:

$$
\tilde{\mathbf{C}}_{0}^{\mathrm{T}} \Delta \mathbf{e}_{1}-\tilde{\mathbf{C}}_{0}^{\mathrm{T}} \mathbf{Q}_{1}^{\mathrm{T}} \Delta \mathbf{d}-\Delta \mathbf{e}_{0}+\mathbf{Q}_{0}^{\mathrm{T}} \Delta \mathbf{d}=\mathbf{0} .
$$

Introducing the expressions of $\Delta \mathbf{e}_{0}$ and $\Delta \mathbf{e}_{1}$ given by Eqs. (43) into (45) and using (36) we obtain:

$$
\mathbf{R}^{\mathrm{T}} \mathbf{F} \mathbf{R} \Delta \mathbf{s}_{0}+\mathbf{R}^{\mathrm{T}} \mathbf{F} \Delta \mathbf{v}-\mathbf{R}^{\mathrm{T}} \mathbf{Q}^{\mathrm{T}} \Delta \mathbf{d}+\sum_{j=1}^{n_{\mathrm{cp}}}\left[\mathbf{R}^{\mathrm{T}} \mathbf{N}_{\sigma}^{\mathrm{T}}\left(\mu \bar{g}^{2}+\lambda \bar{g}\right) \nabla_{\sigma} \bar{g}\right]_{j}=\nabla_{\Delta \mathbf{s}_{0}} \Omega_{\mathrm{c}}^{\mathrm{AL}}=\mathbf{0}
$$

which is the expression of the gradient w.r.t. $\Delta \mathbf{s}_{0}$ of the discretised functional $\Omega_{\mathrm{c}}^{\mathrm{AL}}$ (Eq. (39)): at the solution it is equal to zero.

\section{Iterative solution scheme}

In this section we will describe the numerical procedure employed to obtain the solution of the incremental problem (40). The procedure closely follows the one described in Cuomo and Ventura (1998) and is a particularisation to the problem at hand of the method described in Bertsekas (1982) and Fletcher (1987).

The solution is obtained in two steps, iterating independently on the direct and dual variable sets, given, respectively, by the stress and thermodynamic force increments and by the plastic multipliers. Optimisation w.r.t. a set of variables is achieved keeping the values of the variables of the other set fixed. As the constrained function is convex on the whole direct variable space, the generalised complementary energy functional $\Omega_{\mathrm{c}}\left(\Delta \mathbf{s}_{0}, \Delta \mathbf{c}\right)$ is also convex; moreover, as $\mu>0$ and the Lagrangian multipliers are non negative by the Kuhn and Tucker conditions, the Augmented Lagrangian functional $\Omega_{\mathrm{c}}^{\mathrm{AL}}\left(\Delta \mathbf{s}_{0}, \Delta \mathbf{c}, \lambda\right)$, Eq. (39), is also convex w.r.t. $\left(\Delta \mathbf{s}_{0}, \Delta \mathbf{c}\right)$ for any penalty parameter value (Bertsekas, 1982) and concave w.r.t. $\lambda$.

In the numerical implementation the non-negativity of the Lagrangian multipliers is guaranteed if the procedure is initialised at each stage by a Lagrangian multiplier update. Subsequently, the optimal values of the direct variables are sought with a classical Newton iteration scheme, usually using the following elastic solution as the trial value:

$$
\Delta \mathbf{s}_{0}=\left(\mathbf{R}^{\mathrm{T}} \mathbf{F R}\right){ }^{1} \mathbf{R}^{\mathrm{T}}\left[\mathbf{Q}^{\mathrm{t}} \Delta \mathbf{d}-\mathbf{F} \Delta \mathbf{v}\right] ; \quad \Delta \mathbf{c}=0 .
$$

The refinement of the solution $\Delta \mathbf{x}=\left(\Delta \mathbf{s}_{0}, \Delta \mathbf{c}\right)$ at the next step 


$$
\Delta \mathbf{x}_{k+1}=\Delta \mathbf{x}_{k}-\left(\nabla_{\Delta \mathbf{x} \Delta \mathbf{x}}^{2} \Omega_{\mathrm{c}}^{\mathrm{AL}}\right)_{k}{ }^{1}\left(\nabla_{\Delta \mathbf{x}} \Omega_{\mathrm{c}}^{\mathrm{AL}}\right)_{k}
$$

requires evaluation of the Hessian of the Augmented Lagrangian functional.

The Lagrangian multipliers are updated keeping the value of the direct variables fixed. Several multiplier update formulas can be used, depending on the iterative scheme adopted for the dual problem. First- or second-order formulas have been proposed, according to whether a steepest ascent optimisation or a Newton-like technique is applied. A deeper examination of these alternatives, as well as of the numerical iterative schemes and their physical meaning is to be found in Cuomo et al. (1998). In the present paper the first-order Hestenes-Powell update formula (Hestenes, 1969; Powell, 1969) will be used:

$$
\lambda_{j}^{i+1}=\lambda_{j}^{i}+\Delta \lambda_{j}^{i} \quad \Delta \lambda_{j}^{i}=\mu g_{j}\left(\mathbf{s}_{0}+\Delta \mathbf{s}_{0}^{i}, \mathbf{c}+\Delta \mathbf{c}^{i}\right)
$$

where $j$ denotes the $j$-th check point.

The direct and dual optimisations are performed sequentially until the constraints are met. This is achieved when an appropriate norm of the indicator function of the domain $K$ is reduced to zero. It has been found that good convergence is achieved using the control:

$$
\max _{j=1, n_{\mathrm{cp}}}\left|\left(\frac{1}{2} \mu \bar{g}^{2}+\lambda \bar{g}\right)\right|_{j}<\gamma
$$

where $\gamma$ is a fixed tolerance.

\section{Numerical tests}

The algorithm described in the previous sections was implemented in an original code. Linear and non linear isotropic and kinematic hardening models were used in various combinations.

The computational efficiency of the algorithm was verified with reference to the classical example of the Cook membrane, the results of which are widely known in literature (Simo et al., 1989; Weissman and Jamjian, 1993). The geometrical and mechanical characteristics are given in Fig. 5.

The beam is clamped at one end and is acted upon by a permanent $q_{\text {per }}=0.0625 \mathrm{KN} / \mathrm{mm}$ and a variable $\rho q_{\text {acc }}=\rho 0.0625 \mathrm{KN} / \mathrm{mm}$ tangential load distributed over the free end; $\rho$ is a scalar in the range $\rho \in[0.1,0.8]$. The analysis was performed in plane-stress $\mathbf{J}_{2}$ plasticity with isotropic, kinematic and mixed hardening and in perfect plasticity. The following material properties were used:

- Young's modulus $E=70 \mathrm{KN} / \mathrm{mm}^{2}$;

- Poisson's ratio $v=1 / 3$;

- uniaxial tensile yield stress $\sigma_{0}=0.243 \mathrm{KN} / \mathrm{mm}^{2}$;

- linear isotropic hardening modulus $H_{\text {iso }}=0.2 \mathrm{KN} / \mathrm{mm}^{2}$;

- linear kinematic hardening modulus $H_{\mathrm{kin}}=0.015 \mathrm{KN} / \mathrm{mm}^{2}$;

- non linear isotropic hardening modulus $H_{\text {iso }}=0.135 \mathrm{KN} / \mathrm{mm}^{2}$;

- mixed linear hardening moduli $H_{\text {iso }}=0.135 \mathrm{KN} / \mathrm{mm}^{2}, H_{\text {kin }}=0.015 \mathrm{KN} / \mathrm{mm}^{2}$.

The membrane was discretised into an equal number of elements in the horizontal and vertical directions. 4-node bilinear elements with one control point for plastic admissibility at their centre and 8node serendipity elements with four control points were used; the number of elements per side ranged from 2 to 20.

The permanent load, equivalent to a resultant force of $1.0 \mathrm{KN}$, corresponds to an elastic state. 


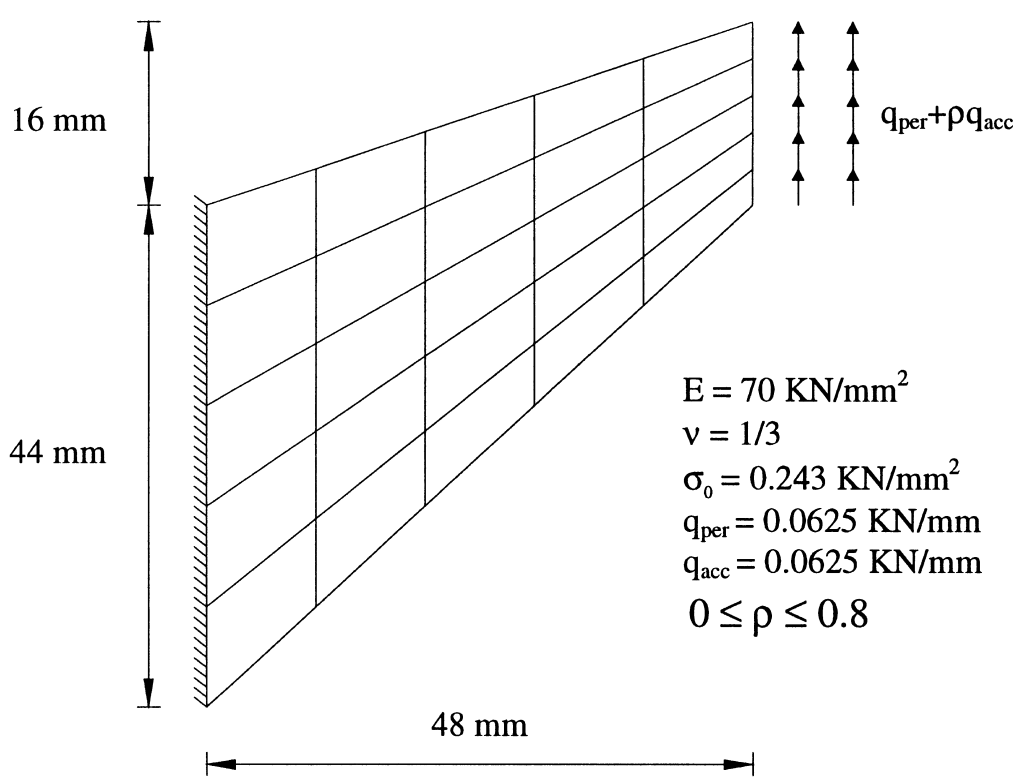

Fig. 5. Tapered beam: geometrical and mechanical characteristics.

Successively, the load is increased with step of $\rho=0.1$ up to a final value of $F=F_{\text {per }}+\rho F_{\text {acc }}=1.8 \mathrm{KN}$. When $\rho=0.2$ yielding first occurs at the bottom right-hand corner. Whit $\rho=0.8$ the beam is almost fully plasticised except for a diagonal axis, as shown in Fig. 6. The relevant deformed shape is illustrated in Fig. 7.
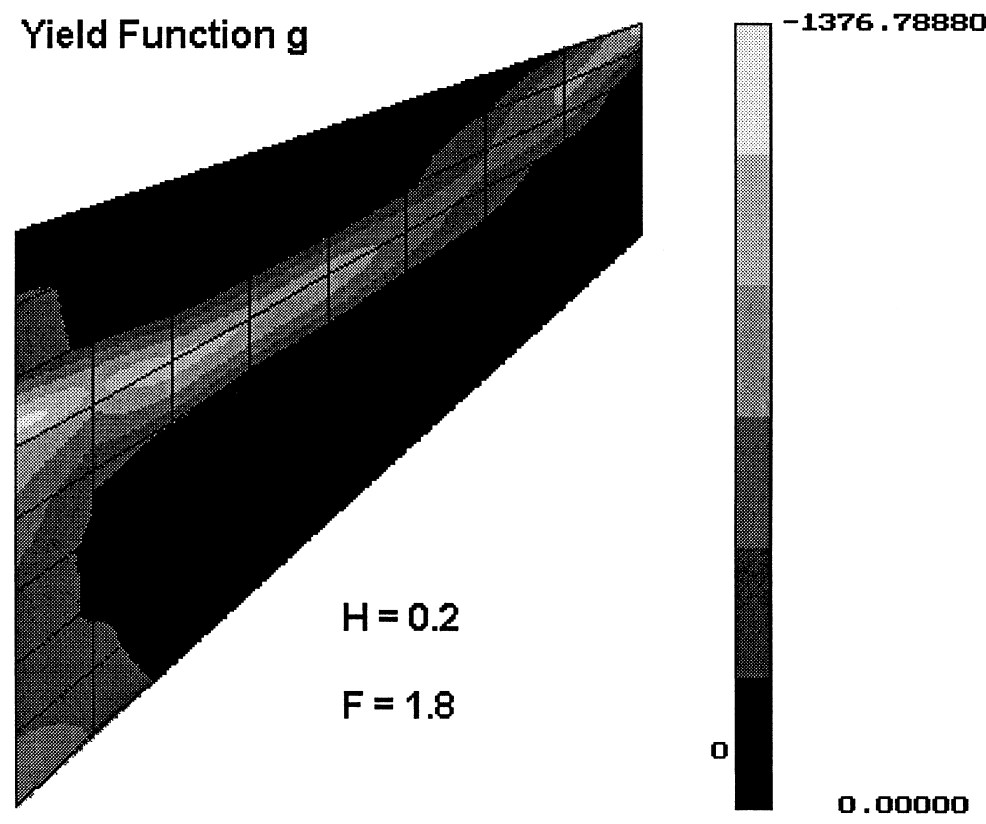

Fig. 6. Tapered beam: Yield function for $F \quad 1.8$. 

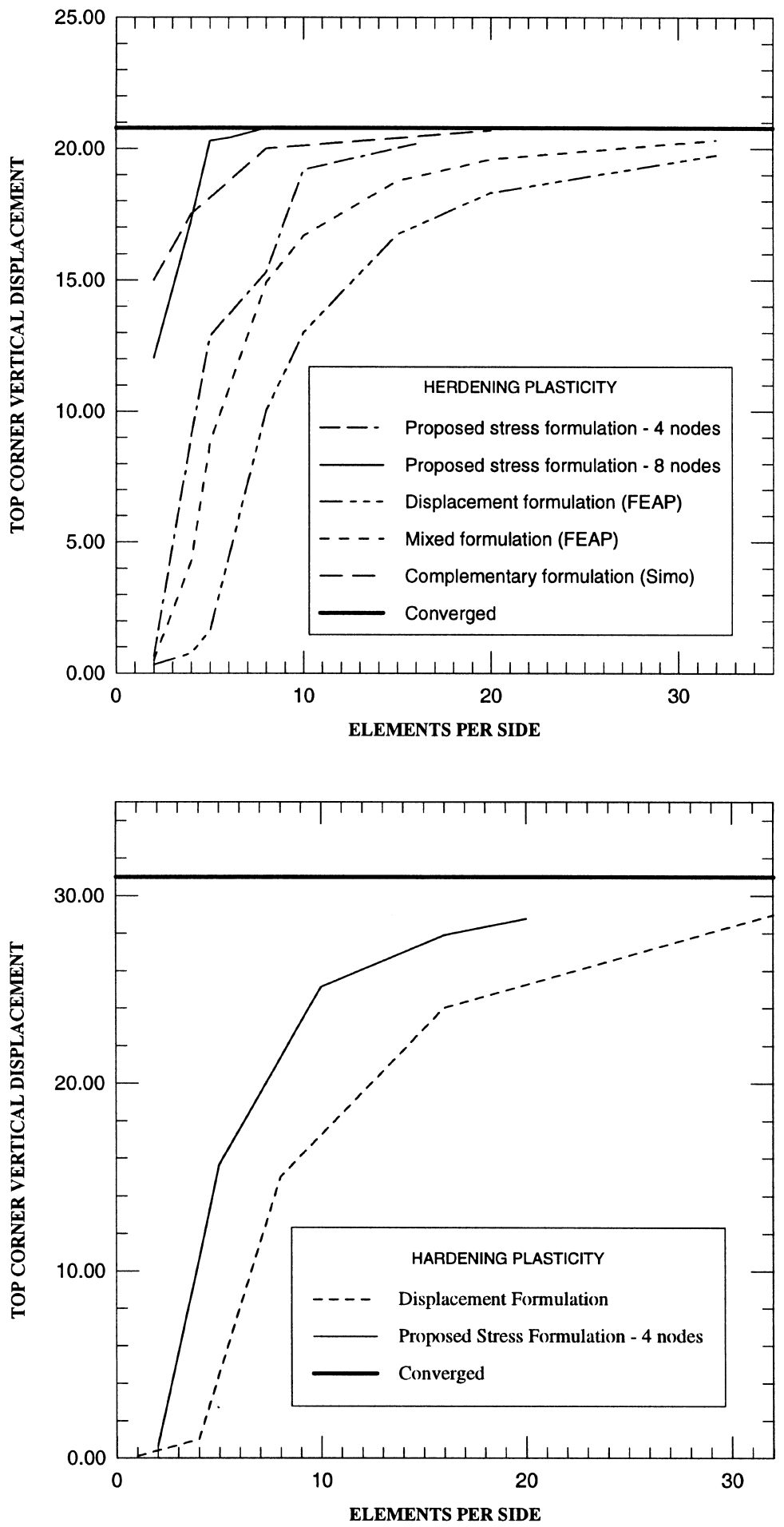

Fig. 8. (a) Tapered beam: Convergence for the proposed stress formulation compared with other models. Linear isotropic harden ing. $H_{\text {iso }} 0.2 \mathrm{KN} / \mathrm{mm}^{2}$. (b) Tapered beam: Convergence for the proposed stress formulation and the displacement model. Mixed hardening. $H_{\text {iso }} \quad 0.135 \mathrm{KN} / \mathrm{mm}^{2}, H_{\text {kin }} \quad 0.015 \mathrm{KN} / \mathrm{mm}^{2}$. 


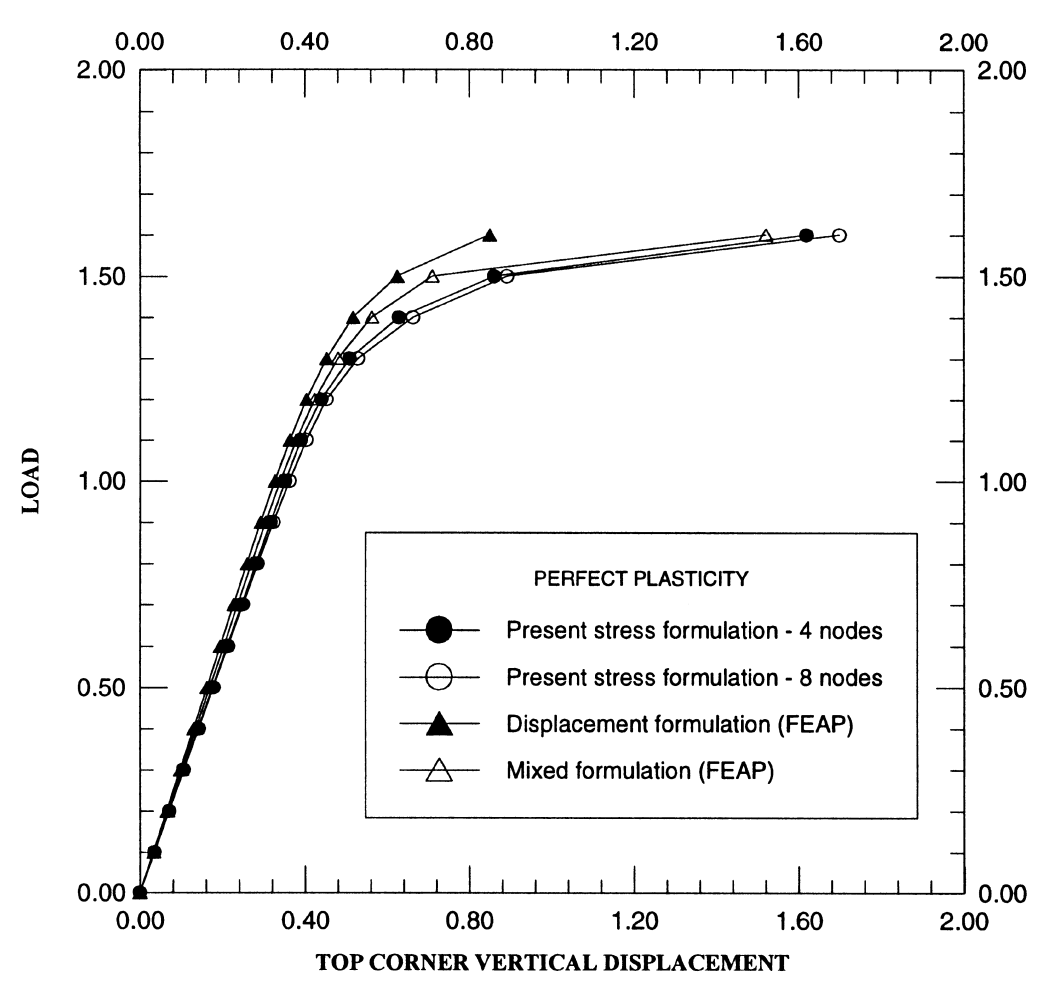

Fig. 11. Tapered beam (64 elements): Displacement load curves for perfect plasticity.

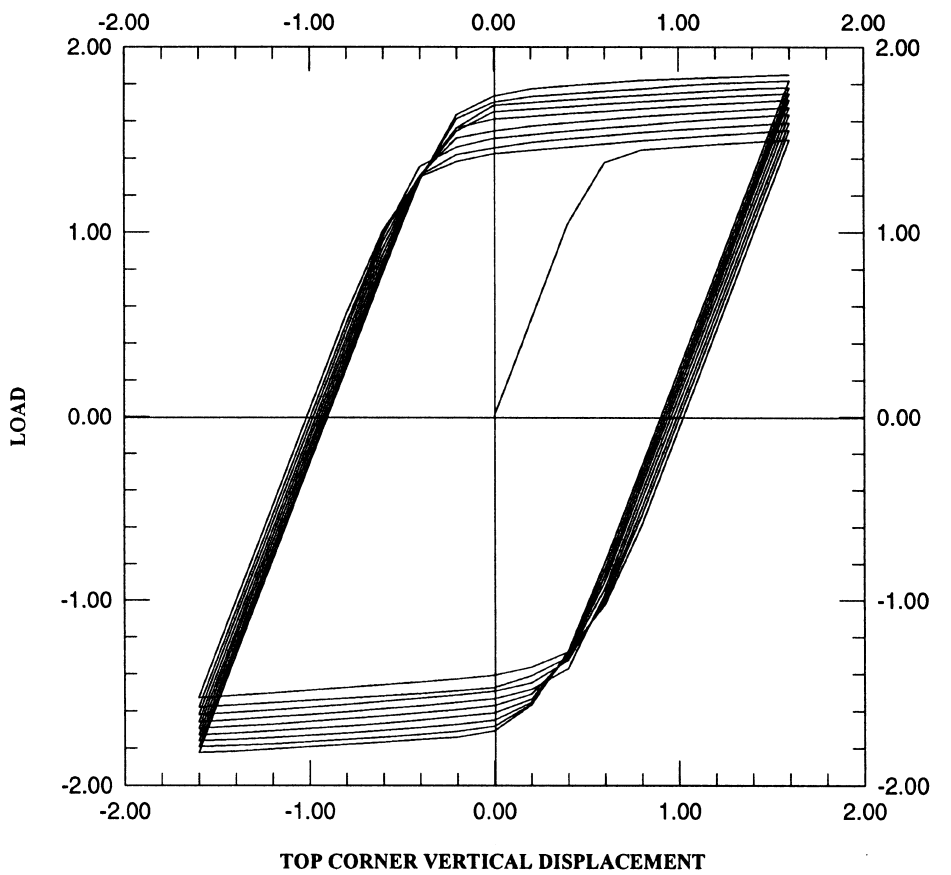

Fig. 12. Top corner nodal vertical force for cyclic imposed displacement. Linear isotropic hardening. $H_{\text {iso }} \quad 0.2 \mathrm{KN} / \mathrm{mm}^{2}$. 


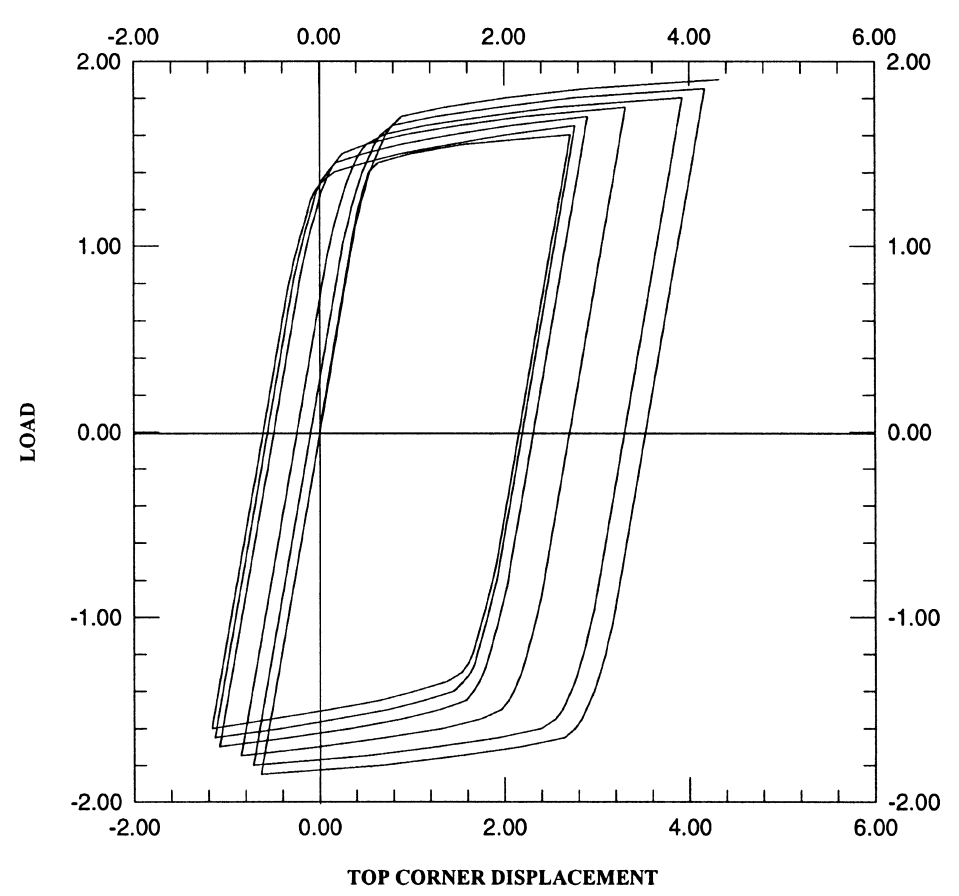

Fig. 15. Top corner vertical displacement for cyclic load conditions. Mixed hardening. $H_{\text {iso }} 0.135 \mathrm{KN} / \mathrm{mm}^{2}, H_{\text {kin }} 0.015$ $\mathrm{KN} / \mathrm{mm}^{2}$.

\section{Conclusions}

The constitutive laws of elasto-plasticity with internal variables have been revisited through the tools of convex analysis and generalised potentials. According to the generalised standard material hypothesis of Halphen and Nguyen, for reversible behaviour the Helmoltz specific free energy functional has been assumed as the sum of two lower semicontinuous convex potentials while a dissipation functional defines the irreversible phase. The conjugate functionals in the rates of static variables have been derived, analysing particular hardening models.

The rate forms of the generalised $\mathrm{Hu}-$ Washizu, Hellinger-Reissner, Prager-Hodge and Greenberg functionals for inelastic problems and some finite-step formulations have been derived, focusing on a complementary energy formulation that is especially useful in particular structural problems such as those where the displacement field is undetermined. It is also convenient because the admissibility constraint for the internal stress state is directly imposed on the primary variables. The functional, defined over the sets of the thermodynamic forces and stress rates, turns out to be convex, lower semicontinuous, non-smooth and non-differentiable due to the presence of the indicator function for the plastically admissible stress states. This has been regularised through the introduction of the Augmented Lagrangian term.

In the numerical solution the discretised problem has been reformulated in a finite-step form using a fully implicit integration scheme, and the functional has been redefined in the space of the selfequilibrated nodal stress increments, automatically satisfying the equilibrium equations in the weak form and consistently reducing the number of variables. The solution is obtained by iterating independently 
on the direct variables with a classical Newton iteration scheme and updating the dual variables through the first-order Hestenes-Powell formula.

The results of numerical experiments on the classical example of the Cook membrane have shown that the algorithm performs better than classical displacement and mixed methods. It is also equally applicable to perfect or hardening plasticity.

Several issues have arisen from numerical tests, like the necessity of a reduced number of control points in the elements to avoid lack of convergence. Similarly, the Lagrangian term should be correctly integrated in the distribution sense.

The numerical efficiency of the method is, however, limited by the reduction strategy adopted to obtain the self-equilibrated stresses, which leads to sparse matrices. The analysis of tactics to improve the computational efficiency will be the subject of a subsequent paper.

\section{References}

Berkovic, M., Mijuca, D. 1998. An efficient continuous stress mixed model based on the Reissners's principle. In: Idelsohn, S., Oñate, E., Dvorkin, E. (Eds.), Computational Mechanics. CIMNE, Barcelona, Spain.

Bertsekas, D.P., 1982. Constrained Optimization and Lagrange Multiplier Methods. Academic Press, Boston.

Cuomo, M., Contrafatto, L., Ventura, G. 1997. Comparison of some numerical algorithms based on augmented Lagrangian regularisation for elastic plastic analysis. In: Owen, D.R.J., Oñate, E., Hinton, E. (Eds.), Computational Plasticity. Pineridge Press, Barcelona, pp. 20332038.

Cuomo, M., Contrafatto, L., Ventura, G., 1998. Numerical analysis of augmented Lagrangian algorithms applied to a complementary formulation in elastoplasticity with internal variables. International Journal of Numerical Methods in Engineering. Submitted for publication.

Cuomo, M., Ventura, G., 1998. Complementary energy approach to contact problems based on consistent Augmented Lagrangian formulation. Mathematical and Computer Modelling 28 (48), 185204.

Eve, R.A., Reddy, B.D., Rockafellar, R.T., 1990. An internal variable theory of elastoplasticity based on the maximum plastic work inequality. Quarterly of Applied Mathematics 68 (1), 5983.

Fletcher, R., 1987. Pratical Methods of Optimization. Wiley, New York.

Glowinski, R., Le Tallec, P., 1989. SIAM, Philadelphia.

Halphen, B., Nguyen, Q.S., 1975. Sur les matériaux standards généralisés. Journal de Mécanique 14, 3963.

Hestenes, M.R., 1969. Multiplier and gradient methods. Journal of Optimization Theory and Applications 4, 303320.

Olson, M.D. 1983. The mixed finite element method in elastic contact problems. In: Atluri, S.N., Callagher, R.H., Zienkiewicz, O.C. (Eds.), Hybrid and Mixed Element Methods. Wiley, New York, pp. 1949.

Pian, T.H.H., Sumihara, K., 1984. Rational approach for assumed stress finite elements. International Journal of Numerical Methods in Engineering 20, 16851695.

Powell, M.J., 1969. A Method for Non Linear Constraint in Optimization Problems. Academic Press, London.

Rockafellar, R.T., 1970. Convex Analysis. Princeton University Press, Princeton.

Romano, G., Alfano, G. 1995. Variational principles, approximations and discrete formulations in plasticity. In: Owen, D.R.J., Oñate, E., Hinton, E. (Eds.), Computational Plasticity. Pineridge Press, Barcelona, pp. 7182.

Romano, G., Rosati, L., Marotti de Sciarra, F., 1993. A variational theory for finite step elasto plastic problems. International Journal of Solids and Structures 30, 23172334.

Simo, J.C., Kennedy, J.G., Taylor, R.L., 1989. Complementary mixed finite element formulations for elastoplasticity. Computer Methods in Applied Mechanics and Engineering 74, 177206.

Simo, J.C., Rifai, M.S., 1990. A class of mixed assumed strain methods and the method of incompatible modes. International Journal of Numerical Methods in Engineering 29, 15951638.

Simo, J.C., Taylor, R.L., 1985. Consistent tangent operators for rate independent elastoplasticity. Computer Methods in Applied Mechanics and Engineering 48, 101118. 
Taylor, R.L., 1998. A Finite Element Analysis Program - Version 6.4b.

Weissman, S.L., 1992. A mixed formulation of non linear-elastic problems. Computers and Structures 44 (4), 813-822.

Weissman, S.L., Jamjian, M., 1993. Two-dimensional elastoplasticity: approximation by mixed finite elements. International Journal of Numerical Methods in Engineering 36, 3703-3727.

Zienkievicz, O.C., Taylor, R.L., 1989. The Finite Element Method, 4th ed. McGraw-Hill, London. 